\title{
Interior Tomography Using 1D Generalized Total Variation. Part II: Multiscale Implementation*
}

\author{
Minji Lee ${ }^{\dagger}$, Yoseob Han ${ }^{\dagger}$, John Paul Ward ${ }^{\ddagger}$, Michael Unser ${ }^{\ddagger}$, and Jong Chul Ye
}

\begin{abstract}
To address the classic interior tomography problem where projections at each view extend only to the shadow of a circular region completely interior to the subject being scanned, previously we showed that the exact recovery of two- and three-dimensional piecewise smooth images is guaranteed using a one-dimensional generalized total variation seminorm penalty which allows a much faster reconstruction. To further accelerate the algorithm up to a level for clinical use, this paper proposes a novel multiscale reconstruction method by exploiting the Bedrosian identity of the Hilbert transform. More specifically, we show that the high frequency parts of the one-dimensional signals can be quickly recovered analytically with the Hilbert transform because of the Bedrosian identity. This implies that computationally expensive iterative reconstruction need only be applied to low resolution images in the downsampled domain, which significantly reduces the computational burden. Moreover, even for incomplete trajectories such as circular cone-beam geometry, we demonstrate that the proposed multiscale interior tomography approach can be combined with a novel spectral blending method in order to mitigate cone-beam artifacts from missing frequency regions. We show the efficacy of the proposed multiscale algorithm using circular fan-beam, helical cone-beam data, and circular conebeam geometry. With a graphics processing unit implementation, we demonstrate that the speed of the algorithm can be significantly accelerated up to the level for clinical use for various acquisition geometries.
\end{abstract}

Key words. interior tomography, Hilbert transform, generalized TV, Bedrosian theorem, multiscale decomposition, spectral blending

AMS subject classifications. $44 \mathrm{~A} 12,44 \mathrm{~A} 15$

DOI. $10.1137 / 15 \mathrm{M} 1015881$

1. Introduction. The aim of this paper is to address the classical interior problem, which is a limited data sampling problem from a two-dimensional (2D) or three-dimensional (3D) $\mathrm{x}$-ray transform where projections at each view extend only to the shadow of a circular region completely interior to the subject being scanned. Our object model is continuous, and the view angle and detector sampling are also continuous. For example, Figure 1 illustrates a 2D circular fan-beam source and detector systems with two types of detector sizes, where the region of interest (ROI) area of each case is shaded in gray. While the detector in Figure 1(a) has a large enough size to cover the whole support of the object, the detector in Figure 1(b)

\footnotetext{
${ }^{*}$ Received by the editors April 8, 2015; accepted for publication (in revised form) August 31, 2015; published electronically October 27, 2015. This work was supported by the Samsung Advanced Institute of Technology, Samsung Electronics, and the Korea Science and Engineering Foundation under grant NRF-2014R1A2A1A11052491.

http://www.siam.org/journals/siims/8-4/M101588.html

${ }^{\dagger}$ Bio Imaging \& Signal Processing Laboratory, Department of Bio and Brain Engineering, Korea Advanced Institute of Science \& Technology (KAIST), Yuseong-gu, Daejon 305-701, Korea (minjilee@kaist.ac.kr, hanyoseob@kaist.ac.kr, jong.ye@kaist.ac.kr).

${ }^{\ddagger}$ Biomedical Imaging Group, École Polytechnique Fédérale de Lausanne (EPFL), CH-1015 Lausanne, Switzerland (john.ward@epfl.ch, michael.unser@epfl.ch).
} 


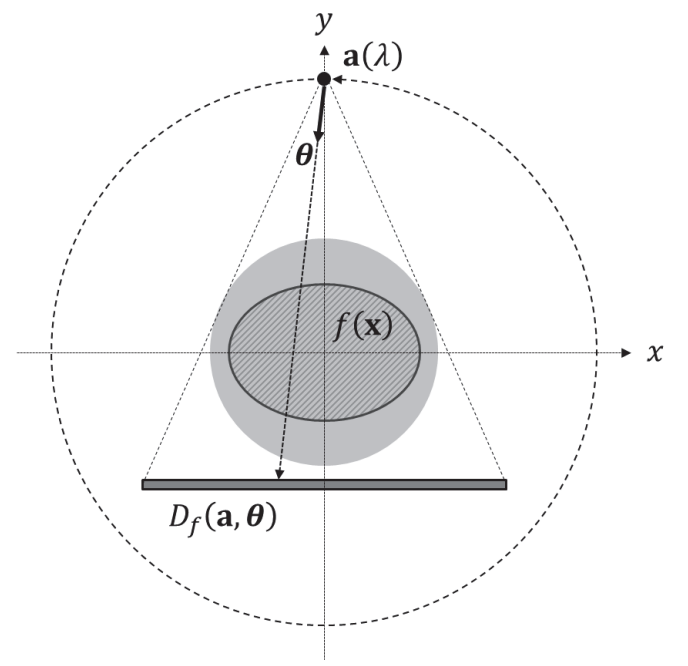

(a) Full-beam tomography

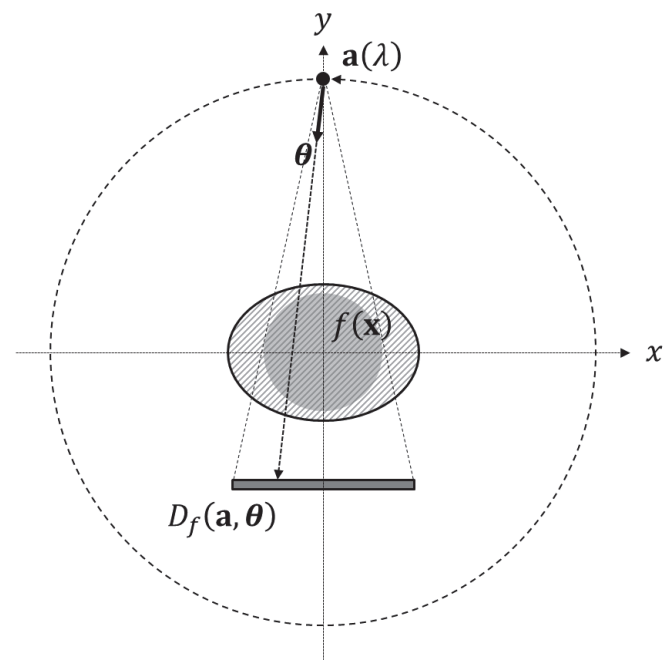

(b) Interior tomography

Figure 1. Full-beam and interior tomography in a $2 D$ circular fan-beam geometry.

is too small to cover the object from any projection view. We call the case in Figure 1(b) interior tomography or ROI tomography.

Interior tomography is useful in reducing the radiation exposure by limiting the x-ray aperture for specific organs such as the heart. It has also been investigated to reduce the x-ray detector size for cost savings. However, due to detector truncation at each view, the underlying image cannot be reconstructed accurately by analytic methods, and approximate methods such as sinogram extrapolation method [14] are widely used instead. Nonetheless, this often introduces bias to the $\mathrm{CT}$ number in the reconstructed image.

So far, most of the prior studies $[5,8,22,36,37,38,40]$ have been concerned about ROI reconstruction problems where the projection data at some view angles are not truncated, so one can use differentiated backprojection (DBP) to reconstruct the entire ROI starting from the complete data region. On the other hand, due to detector truncation at all projection views, our problem is more restricted, and these approaches cannot directly be used. Recently, it was shown that an ROI image can be reconstructed uniquely from truncated projection data when the intensity of the subregions inside the ROI is known a priori [6]. However, in general, it is difficult to know the intensity inside an object. In [15], a continuous domain singular value decomposition of the finite Hilbert transform operator characterized in [17] was used to represent an interior image with a linear combination of the eigenfunctions, after which the null space compensation was performed by using a more general set of prior subregion knowledge. Moreover, Katsevich, Katsevich, and Wang [18] proved the general uniqueness results for the interior problem and provided stability estimates. On the other hand, using 2D or $3 \mathrm{D}$ total variation (TV), the authors in $[13,34,35]$ showed that a unique reconstruction is possible if the images are piecewise constant, linear, or smooth. In these works, an iterative procedure to reconstruct the interior images requires multiple applications of forward and backward projections; thus, its computational complexity prohibits its routine use clinically,

Copyright (c) by SIAM. Unauthorized reproduction of this article is prohibited. 
even though highly optimized implementations of these iterative approaches are currently available for clinical use [27].

In our previous work [32], we revisited the classical interior problem, where projections at each view extend only to the shadow of a circular region completely interior to the subject being scanned, and the object model is continuous, and the view angle and detector sampling are also continuous. In particular, we were also interested in reconstructing images that are piecewise smooth; i.e., the domain where the image is defined can be decomposed into a finite number of subdomains such that the image is a smooth function on each piece. However, our approach generalized the ideas developed in [13, 34, 35] with a simplified one-dimensional (1D) formulation on the chord lines, and as a result, we were able to perfectly reconstruct new classes of functions. In particular, we showed that we can reconstruct any image that is a generalized L-spline along a collection of chord lines passing through the ROI [32]. For example, we can reconstruct images whose 1D restrictions are nonpolynomial exponential B-splines, which were not covered in [13, 34, 35].

To further accelerate the algorithm up to a level for clinical use, this paper proposes a multiscale reconstruction method that sequentially reconstructs the low frequency and high frequency parts of a 1D signal at different resolutions. Related frequency-split reconstruction methods have been used in the x-ray CT community for various purposes, such as artifact reduction from redundant data [28] or metal artifact reduction [20]. However, in our case, the frequency-split is a natural by-product of the Hilbert transform property, which can be used for a more efficient numerical implementation. More specifically, we show that the high frequency part of the signal can be recovered analytically using a one-step Hilbert transform thanks to the powerful Bedrosian identify in the Hilbert transform of the product of two functions [19]. Therefore, computationally expensive iterative reconstruction need only be performed to reconstruct the low frequency part of the signal after downsampling, which enables a fast implementation. In addition, the algorithm can be performed in parallel for each chord, which lends itself for easy implementation using a graphics processing unit (GPU).

Yet, there are many CT acquisition trajectories which are not complete in terms of Tuy's condition [31]. For example, circular cone-beam CT has been widely used in practice because the trajectory can be easily implemented in hardware, but the trajectory is not complete, and the chord lines are not defined for whole slices of an object, except for the midplane. This results in cone-beam artifacts as the cone angle increases. Accordingly, the direct application of the proposed multiscale implementation to a circular cone-beam trajectory is not feasible. To address these technical difficulties, we show that the DBP data on a virtual chord line [36] can also be represented as a 1D Hilbert transform of an underlying signal that is filtered through a spatially varying channel with the missing frequency regions. Therefore, to reduce the cone-beam artifacts, we extend the Fourier blending idea proposed for the half-scan filtered backprojection (FBP) algorithm [25] to our multiscale interior tomography approach and provide a novel Fourier domain two-way weighting scheme. The new weighting scheme uses a bow tie window that combines the multiscale interior tomography results from the horizontal and vertical directions.

We verify the efficacy of the algorithm using various acquisition trajectories, which show that a very accurate reconstruction can be obtained quickly on a practical level using the clinical helical or circular cone-beam CT acquisition geometry.

Copyright (c) by SIAM. Unauthorized reproduction of this article is prohibited. 
This paper is organized as follows. Section 2 provides the mathematical preliminaries that are the essential components for a 1D formulation of the interior tomography problem. Section 3 describes the main idea of the proposed algorithm, and the experimental results are presented in section 4 . In section 5, we discuss the results in detail and conclude in section 6 .

2. Mathematical preliminaries. In this section, we provide the mathematical preliminaries and review the main idea of our previous work [32].

2.1. Fourier analysis of DBP from a complete trajectory. The main part of this subsection is not novel because it has already appeared in $[23,38,39]$ to derive the backprojection filtration (BPF) algorithms. Nevertheless, we repeat it here for self-inclusiveness.

Specifically, for a given object function $f(\mathbf{x})$, its $d$-dimensional inverse Fourier transform from the spectrum $\hat{f}(\boldsymbol{\omega})$ is given by

$$
f(\mathbf{x})=\frac{1}{(2 \pi)^{d}} \int d \boldsymbol{\omega} \hat{f}(\boldsymbol{\omega}) e^{j \mathbf{x} \cdot \boldsymbol{\omega}}
$$

where $d=2,3$ are for the 2D and 3D tomography problems, respectively. In addition, if $\boldsymbol{\theta}$ denotes the $d$-dimensional unit vector and $\mathbf{a} \in \mathbb{R}^{d}$ is the source location, then the x-ray transform $D_{f}$ maps a function on $\mathbb{R}^{d}$ into the set of its line integrals

$$
D_{f}(\mathbf{a}, \boldsymbol{\theta})=\int_{0}^{\infty} d t f(\mathbf{a}+t \boldsymbol{\theta})
$$

For a given object function $f(\mathbf{x})$ and a given source trajectory $\mathbf{a}(\lambda), \lambda \in\left[\lambda_{\min }, \lambda_{\max }\right]$, we now define the DBP:

$$
g(\mathbf{x})=\left.\int_{\lambda^{-}}^{\lambda^{+}} d \lambda \frac{1}{\|\mathbf{x}-\mathbf{a}(\lambda)\|} \frac{\partial}{\partial \mu} D_{f}(\mathbf{a}(\mu), \boldsymbol{\theta})\right|_{\mu=\lambda},
$$

where $\left[\lambda^{-}, \lambda^{+}\right] \subset\left[\lambda_{\min }, \lambda_{\max }\right]$ denotes the appropriate intervals from the source segments between $\lambda_{\min }$ and $\lambda_{\max }$, and $1 /\|\mathbf{x}-\mathbf{a}(\lambda)\|$ denotes the distance weighting. Then, the following theorem holds.

Theorem 2.1. Let the source trajectory $\mathbf{a}(\lambda), \lambda \in\left[\lambda^{-}, \lambda^{+}\right]$have no discontinuities. Suppose, furthermore, $\mathbf{x}$ is on the connecting line of the two source positions $\mathbf{a}\left(\lambda^{-}\right)$and $\mathbf{a}\left(\lambda^{+}\right)$. Then, the DBP data in (2.2) can be represented as

$$
g(\mathbf{x})=\frac{1}{(2 \pi)^{d}} \int d \boldsymbol{\omega} \hat{f}(\boldsymbol{\omega}) e^{j \mathbf{x} \cdot \boldsymbol{\omega}} j \sigma\left(\mathbf{x}, \boldsymbol{\omega}, \lambda^{-}, \lambda^{+}\right)
$$

where

$$
\begin{gathered}
\sigma\left(\mathbf{x}, \boldsymbol{\omega}, \lambda^{-}, \lambda^{+}\right)=\frac{1}{2}\left[\operatorname{sgn}\left(\boldsymbol{\alpha}\left(\lambda^{-}, \mathbf{x}\right) \cdot \boldsymbol{\omega}\right)-\operatorname{sgn}\left(\boldsymbol{\alpha}\left(\lambda^{+}, \mathbf{x}\right) \cdot \boldsymbol{\omega}\right)\right], \\
\boldsymbol{\alpha}(\lambda, \mathbf{x})=\frac{\mathbf{x}-\mathbf{a}(\lambda)}{\|\mathbf{x}-\mathbf{a}(\lambda)\|} .
\end{gathered}
$$

Proof. It can be derived from a result in [23, 38, 39]. 


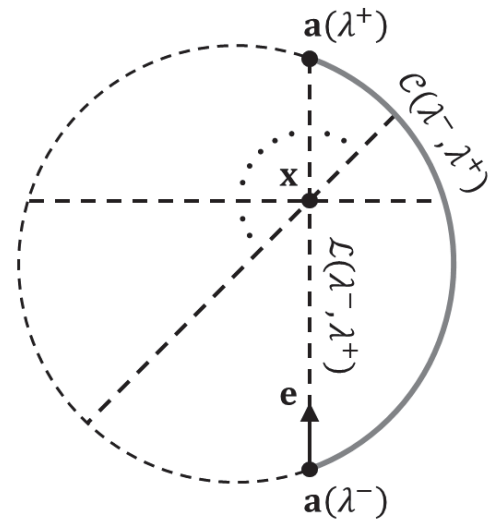

(a)

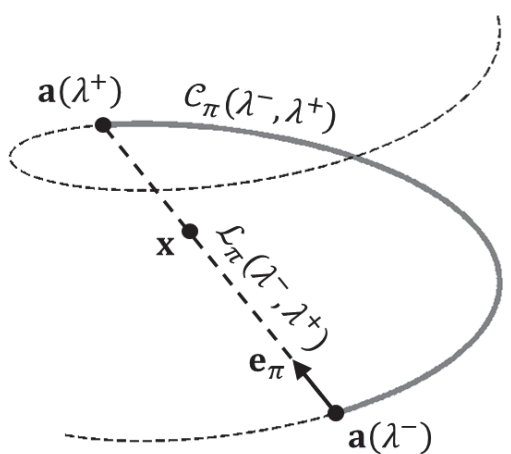

(b)

Figure 2. Illustration of the chord line and the PI line. (a) is for the chord line in the circular trajectory, and (b) is for the PI line in the helical trajectory.

The connecting line between the two sources position is often called a chord line $[23,38,39]$. If the unit vector $\mathbf{e}$ along the chord line (in particular, along the directional vector $\boldsymbol{\alpha}\left(\lambda^{-}, \mathbf{x}\right)$ ) is set as a coordinate axis, then we can find the $(d-1)$-dimensional orthonormal basis $E^{\perp}$ such that $V=\left[\mathbf{e}, E^{\perp}\right]$ consists of the basis for the local coordinate system at $\mathbf{a}\left(\lambda^{-}\right)$. Suppose $\left(x^{\prime}, \mathbf{x}_{E^{\perp}}\right) \in \mathbb{R}^{d}$ denotes the coordinate value on the new coordinate system composed of $\mathbf{e}$ and $E^{\perp}$. Then, the authors in $[23,38,39]$ showed that $(2.3)$ can be converted into the following form:

$$
g_{\mathbf{x}_{E \perp}}\left(x^{\prime}\right)=\int \frac{d \eta}{\pi\left(x^{\prime}-\eta\right)} f_{\mathbf{x}_{E \perp}}(\eta):=\mathcal{H}_{\mathbf{e}}\left\{f_{\mathbf{x}_{E \perp}}\left(x^{\prime}\right)\right\}
$$

where $g_{\mathbf{x}_{E \perp}}\left(x^{\prime}\right)$ and $f_{\mathbf{x}_{E \perp}}\left(x^{\prime}\right)$ denote the restriction of $g(\mathbf{x})$ and $f(\mathbf{x})$ on a given $\mathbf{x}_{E^{\perp}}$, respectively, and $\mathcal{H}_{\mathbf{e}}$ denotes the Hilbert transform along the direction vector $\mathbf{e}$ of the chord line. Because $\mathcal{H} \mathcal{H}=-I$, we have $f_{\mathbf{x}_{E^{\perp}}}(x)=-\mathcal{H}_{\mathbf{e}}\left\{g_{\mathbf{x}_{E \perp}}(x)\right\}$, which is known as the BPF method that recovers the object on each chord line by taking the Hilbert transform of the DBP data. The following are already well-known acquisition trajectories that have one or more sets of chord lines over the entire object domain:

- 2D fan-beam CT: In 2D circular fan-beam CT shown in Figure 2(a), the chord line $\mathcal{L}$ that passes through $\mathbf{x}$ inside the circle is not unique; hence, we can choose multiple filtering lines shown in Figure 2(a). For example, a vertical chord line that goes through $\mathbf{x}$ is determined by $\mathbf{a}\left(\lambda^{-}\right)$and $\mathbf{a}\left(\lambda^{+}\right)$. Similarly, we can use horizontal chord lines. The redundancy in the choice of chord lines will be exploited later in mitigating cone-beam artifacts from missing frequency regions in the circular cone-beam trajectory.

- $3 D$ helical cone-beam $C T$ : As shown in Figure 2(b), a helical source trajectory is expressed as

$$
\mathbf{a}(\lambda)=\left(R \cos \lambda, R \sin \lambda, \frac{h}{2 \pi} \lambda\right)^{T} .
$$

Here, $\lambda$ is the rotation angle of the $\mathrm{x}$-ray source which is restricted to $\lambda \in\left[\lambda_{\min }, \lambda_{\max }\right]$, $R$ is the distance from the source to the rotation axis, and $h$ is the pitch of the helix. 


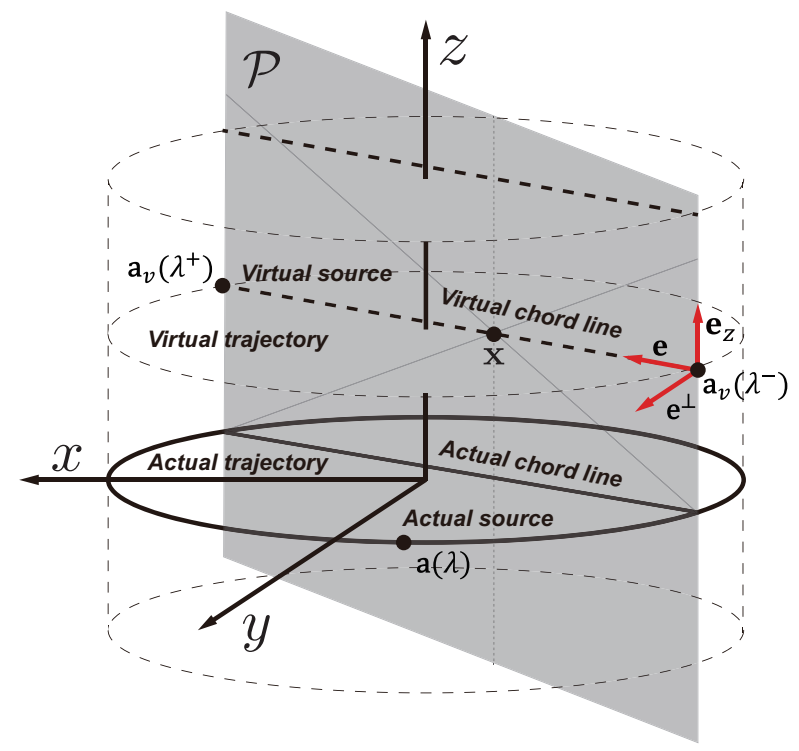

Figure 3. Source trajectories and virtual chord lines. The dark circle is an actual trajectory, and the dark line is an actual chord line. The set of gray-dotted circles is virtual trajectory, and the dark-dot line is a virtual chord line.

The pitch of the helix refers to the translation distance along the $z$-axis during one rotation. In helical cone-beam $\mathrm{CT}$, if two points $\mathbf{a}\left(\lambda^{-}\right), \mathbf{a}\left(\lambda^{+}\right)$on a helical trajectory are selected where $\left|\lambda^{+}-\lambda^{-}\right| \leq 2 \pi$, the chord line connecting these two points denoted as $\mathcal{L}_{\pi}\left(\lambda^{-}, \lambda^{+}\right)$is called a PI line. Each point $\mathbf{x}=(x, y, z)$ in the field of view belongs to one and only one PI line. Then, we can choose our filtering direction as $\mathbf{e}_{\pi}$, the unit vector along the PI line $\mathcal{L}_{\pi}$.

2.2. Fourier analysis of DBP from an incomplete trajectory. Even though the circular cone-beam trajectory is not a complete trajectory, in the following, which is our novel contribution, we will show that a similar Hilbert transform relationship holds true for the virtual chord lines [9], but the frequency content on DBP on the virtual chord line has a missing frequency region. The existence of a missing frequency region in an incomplete trajectory has been well studied [1, 3, 4, 7, 9, 10, 24, 26, 30, 33] since the work by Grangeat [12], and our contribution here is Theorem 2.2, which is essential for deriving the multiscale interior tomography reconstruction algorithm.

The concept of virtual chord lines appeared originally in $[9,36]$ to address circular conebeam reconstruction. Figure 3(a) shows a virtual chord line coordinate system, which is defined by the virtual chord line direction $\mathbf{e}$, the $z$-axis $\mathbf{e}_{z}$, and their perpendicular axis $\mathbf{e}^{\perp}$. The coordinate system consists of both an actual trajectory and a set of virtual trajectory planes for the midplane and the off-midplane, respectively. Using the modified coordinate, a point $\mathbf{x} \in \mathbb{R}^{3}$ in the cylinder is now defined as follows:

$$
\mathbf{x}=\mathbf{a}_{0}+x^{\prime} \mathbf{e}
$$

where $x^{\prime}$ refers to the position of $\mathbf{x}$ along the chord line and the center point of a virtual chord 
line $\mathbf{a}_{0}$; a virtual source location $\mathbf{a}_{v}(\lambda)$ and the direction vector $\mathbf{e}$ of a virtual chord line are defined as follows:

$$
\begin{aligned}
& \mathbf{a}_{0}= \frac{1}{2}\left(\mathbf{a}\left(\lambda^{+}\right)+\mathbf{a}\left(\lambda^{-}\right)\right)+z \mathbf{e}_{z}, \\
& \mathbf{a}_{v}(\lambda)=\mathbf{a}(\lambda)+z \mathbf{e}_{z}, \\
& \mathbf{e}=\frac{\mathbf{a}\left(\lambda^{+}\right)-\mathbf{a}\left(\lambda^{-}\right)}{\left\|\mathbf{a}\left(\lambda^{+}\right)-\mathbf{a}\left(\lambda^{-}\right)\right\|},
\end{aligned}
$$

where $z$ denotes the $z$-coordinate of the point $\mathbf{x}$ in the Cartesian coordinate system. In this virtual chord line system, the Cartesian coordinate $\mathbf{x}=(x, y, z)$ is now converted to a new coordinate $\left(x^{\prime}, y^{\prime}, z\right)$ such that

$$
\mathbf{x}=x^{\prime} \mathbf{e}+y^{\prime} \mathbf{e}^{\perp}+z \mathbf{e}_{z} .
$$

We also denote the corresponding spatial frequency as $\left(\omega_{x^{\prime}}, \omega_{y^{\prime}}, \omega_{z}\right)$. Then, the following theorem shows that a similar Hilbert transform relationship holds true for the virtual chord lines.

Theorem 2.2. Let the source trajectory $\mathbf{a}(\lambda), \lambda \in\left[\lambda^{-}, \lambda^{+}\right]$, have no discontinuities. Suppose, furthermore, position $\mathbf{x}$ is on the virtual chord line that connects the two virtual source positions $\mathbf{a}_{v}\left(\lambda^{-}\right)$and $\mathbf{a}_{v}\left(\lambda^{+}\right)$and has the coordinate values $\left(x^{\prime}, y^{\prime}, z\right)$ on the virtual chord line coordinate system (2.10). Then, the DBP data in (2.2) can be represented as

$$
g(\mathbf{x})=\frac{1}{2 \pi} \int_{-\infty}^{\infty} d \omega_{x^{\prime}} \hat{\phi}\left(\omega_{x^{\prime}}, y^{\prime}, z\right) j \operatorname{sgn}\left(\omega_{x^{\prime}}\right) e^{j \omega_{x^{\prime}} x^{\prime}},
$$

where

$$
\hat{\phi}\left(\omega_{x^{\prime}}, y^{\prime}, z\right):=\frac{1}{(2 \pi)^{2}} \int d \omega_{x^{\prime}} \int_{\omega_{z} \in\left\{\left(\omega_{x^{\prime}}, \omega_{y^{\prime}}, \omega_{z}\right) \notin \mathcal{N}(z)\right\}} d \omega_{z} \hat{f}\left(\omega_{x^{\prime}}, \omega_{y^{\prime}}, \omega_{z}\right) e^{j\left(y^{\prime} \omega_{y^{\prime}}+z \omega_{z}\right)},
$$

and the missing frequency set $\mathcal{N}(z)$ on $z$ is given by

$$
\mathcal{N}(z)=\left\{\left(\omega_{x^{\prime}}, \omega_{y^{\prime}}, \omega_{z}\right) \mid-\frac{1}{x^{\prime}+\sqrt{R^{2}-\left(y^{\prime}\right)^{2}}} \leq \frac{\omega_{x^{\prime}}}{z \omega_{z}} \leq \frac{1}{\sqrt{R^{2}-\left(y^{\prime}\right)^{2}}-x^{\prime}}\right\},
$$

which trivially becomes an empty set when $z=0$.

Proof. See Appendix A.

Let $g_{\mathbf{x}_{E} \perp}\left(x^{\prime}\right)$ and $\phi_{\mathbf{x}_{E \perp}}\left(x^{\prime}\right)$ denote the 1D restriction of $g(\mathbf{x})$ and $\phi(\mathbf{x})$, respectively, for a given $\left(y^{\prime}, z\right)$ coordinate, where $\phi(\mathbf{x})$ denotes the 1D inverse Fourier transform of $\hat{\phi}\left(\omega_{x^{\prime}}, y^{\prime}, z\right)$ along $\omega_{x^{\prime}}$. Since $j \operatorname{sgn}\left(\omega_{x^{\prime}}\right)$ is the Fourier transform of $-1 /\left(\pi x^{\prime}\right)$, (2.11) can be converted into the following:

$$
g_{\mathbf{x}_{E^{\perp}}}\left(x^{\prime}\right)=\int \frac{d \eta}{\pi\left(x^{\prime}-\eta\right)} \phi_{\mathbf{x}_{E^{\perp}}}(\eta):=\mathcal{H}_{\mathbf{e}}\left\{\phi_{\mathbf{x}_{E^{\perp}}}\left(x^{\prime}\right)\right\},
$$

where $\mathcal{H}_{\mathbf{e}}$ denotes the Hilbert transform along the virtual chord line. Therefore, even though $\phi_{\mathbf{x}_{E^{\perp}}}(x)$ in (2.13) is not the same as $f_{\mathbf{x}_{E \perp}}(x)$ in (2.5), the Hilbert transform relationship (2.5) is still valid with respect to $\hat{\phi}(\cdot)$ thanks to $(2.13)$, which is one of the most important findings that allows us to propose a unified multiscale interior tomography approach.

Copyright (C) by SIAM. Unauthorized reproduction of this article is prohibited. 
2.3. Interior tomography using generalized 1D TV. With a slight abuse of notation, in the following, we denote $g(x):=g_{\mathbf{x}_{E \perp}}(x)$ and $f(x)=f_{\mathbf{x}_{E^{\perp}}}(x)$ (or $f(x)=\phi_{\mathbf{x}_{E \perp}}(x)$ for the case of circular cone-beam geometry).

If the DBP data $g(x)$ are available for the support of object $f(x)$ seen in Figure 1(a), the reconstruction can simply be done by performing the inverse Hilbert transform. However, in the case of an interior tomography problem, the detector is truncated, as seen in Figure 1(b); thus the DBP data are only available within the ROI. The main technical difficulty of such a truncated Hilbert transform is the existence of the null space [17]. More specifically, there exists the nonzero $f_{\mathfrak{N u l}}(x)$ such that

$$
\mathcal{H} f_{\mathfrak{N u r}}(x)=0, \quad x \in\left(e_{1}, e_{2}\right)
$$

where $\left(e_{1}, e_{2}\right)$ denotes the ROI. Indeed, $f_{\mathfrak{N} \mathfrak{u l}}(x)$ can be expressed with the appropriate DBP data $\psi(x)$ outside of the ROI such that

$$
f_{\mathfrak{T} \mathfrak{u l}}(x)=-\frac{1}{\pi} \int_{\mathbb{R} \backslash\left(e_{1}, e_{2}\right)} \frac{d x^{\prime}}{x-x^{\prime}} \psi\left(x^{\prime}\right)
$$

Note that the null space signal $f_{\mathfrak{N u r}}(x)$ in $(2.14)$ is differentiable in any order due to the removal of the origin in the integrand. Accordingly, a required interior tomography formulation finds an appropriate regularization term that suppresses the signal belonging to the null space of the truncated Hilbert transform by exploiting the infinite differentiability of $f_{\mathfrak{N u l}}$.

Instead, our previous work [32] introduced a generalized 1D TV penalty minimization as a simplification of multidimensional TV in $[13,34,35]$. For completeness, we repeat the main ideas of [32]. Specifically, we consider a regularization with respect to a Fourier multiplier operator $\mathrm{L}$ that is defined on $L_{2}(\mathbb{R})$ and satisfies two conditions. First, for any interval $E=\left(e_{1}, e_{2}\right) \subset \mathbb{R}$, we require $\mathrm{L}$ to $\operatorname{map} C_{c}^{\infty}(E)$ to $C_{c}(E)$. Second, the null space of $\mathrm{L}$ should consist of entire functions. An example of such an operator is a constant coefficient differential operator

$$
\mathrm{L}:=a_{K} \mathrm{D}^{K}+a_{K-1} \mathrm{D}^{K-1}+\cdots+a_{1} \mathrm{D}+a_{0},
$$

where $K \geq 1$, D denotes the distributional derivative on $\mathbb{R}$, and each $a_{k}$ is a real number. In this example, the finite-dimensional null space consists of linear combinations of exponential functions multiplied by polynomials. For an operator $\mathrm{L}$ and an interval $E \subset \mathbb{R}$, we formally define the generalized TV seminorm

$$
\|f\|_{T V(\mathrm{~L} ; E)}:=\|\mathrm{L} f\|_{L_{1}(E)},
$$

which is valid when $\mathrm{L} f \in L_{1}(E)$. In order to ensure that this seminorm is valid for a larger class of functions, we use the dual formulation

$$
\|f\|_{T V(\mathrm{~L} ; E)}:=\sup _{h \in C_{h}} \int_{E} \mathrm{~L} f(x) h(x) d x
$$

Copyright $\odot$ by SIAM. Unauthorized reproduction of this article is prohibited. 
where $C_{h}=\left\{h \in C_{c}^{\infty}(E),\|h\|_{L_{\infty}} \leq 1\right\}$. Then, the following is the main theoretical result in [32].

Theorem 2.3 (see [32]). Let $f_{0}(x)$ be a generalized L-spline such that

$$
\mathrm{L} f_{0}(x)=\sum_{n=1}^{N} a_{n} \delta\left(x-x_{n}\right)
$$

on E. Then, the minimization problem

$$
\underset{f}{\operatorname{argmin}}\|f\|_{T V(\mathrm{~L} ; E)} \quad \text { subject to } \quad \mathcal{H} f_{0}(x)=\mathcal{H} f(x), \quad x \in E,
$$

has the unique solution equal to $f_{0}(x)$.

2.3.1. Optimization framework. For reconstruction under generalized TV, our previous paper [32] used projections onto convex sets (POCS) algorithm with five constraint sets. Note that this optimization framework is based on continuous formulation in the function space; however, the algorithm can be implemented in a discrete setting for practical applications.

Specifically, the constraint sets $\left\{C_{j}\right\}_{j=1}^{5}$ are given by

$$
\begin{aligned}
& C_{1}=\left\{f(x) \in L^{2}(\mathbb{R}): f(x)=0, x \notin\left(b_{1}, b_{2}\right)\right\}, \\
& C_{2}=\left\{f(x) \in L^{2}(\mathbb{R}): \mathcal{H} f(x)=g(x), x \in\left(e_{1}, e_{2}\right)\right\}, \\
& C_{3}=\left\{f(x) \in L^{2}(\mathbb{R}):\|f\|_{T V(\mathrm{~L} ; E)} \leq \tau\right\}, \\
& C_{4}=\left\{f(x) \in L^{2}(\mathbb{R}): \int_{b_{1}}^{b_{2}} d x f(x)=c_{f}\right\}, \\
& C_{5}=\left\{f(x) \in L^{2}(\mathbb{R}): f(x) \geq 0, x \in\left(b_{1}, b_{2}\right)\right\},
\end{aligned}
$$

where $\left(b_{1}, b_{2}\right)$ denotes support of the object, and $c_{f}$ can be obtained from $D_{f}(\mathbf{a}, \boldsymbol{\theta})$. The additional constraint beyond the necessity in proving the uniqueness in Theorem 2.3 is to make the algorithm converge faster. The optimized solution of $f(x)$ can then be solved by POCS. More specifically, if $P_{C_{j}}$ is the orthogonal projector onto the convex set $C_{j}$ for $j=1, \ldots, 5$, the iteration of the POCS algorithm can be described as

$$
f_{k}(x)=P_{C_{5}} P_{C_{4}} P_{C_{3}} P_{C_{2}} P_{C_{1}} f_{k-1}(x)
$$

with some arbitrary initialization. This algorithm converges to one element of the intersection of the constraints if the intersection is not empty.

Here, the projections to the constraints $C_{1}$ and $C_{5}$ are quite straightforward, and we can just impose the support constraint and the nonnegativity for the intermediate reconstruction. For the projection on the constraints $C_{2}$ and $C_{4}$, we can use the finite inverse Hilbert transform formula [19]. More specifically, we have

$$
f(x)=\frac{1}{\pi} \frac{1}{\sqrt{\left(x_{2}-x\right)\left(x-x_{1}\right)}}\left[\int_{x_{1}}^{x_{2}} d x^{\prime} \frac{1}{x^{\prime}-x} g\left(x^{\prime}\right) \sqrt{\left(x_{2}-x^{\prime}\right)\left(x^{\prime}-x_{1}\right)}+c_{f}\right],
$$

where $x_{1}, x_{2}$ are any points on $\mathcal{L}\left(\lambda^{-}, \lambda^{+}\right)$satisfying $\left(x_{1}, x_{2}\right) \supset\left(b_{1}, b_{2}\right)$.

Copyright (c) by SIAM. Unauthorized reproduction of this article is prohibited. 
Now, the most complicated step is to project on the generalized TV ball. It turns out that the projection on $C_{3}$ from a point $f_{0}$ can be implemented as the following denoising step $[2,11]$ :

$$
\tilde{f}=P_{C_{3}}\left(f_{0}\right)=\underset{f \in C_{f}}{\operatorname{argmin}}\left\{\left\|f-f_{0}\right\|_{2}^{2}+2 \lambda\|f\|_{T V(\mathrm{~L} ; E)}\right\}
$$

for an appropriate Lagrangian parameter. In (2.22), $C_{f}=B_{l, u}$ is a bound constraint:

$$
B_{l, u}=\{x: l \leq x \leq u\}
$$

Using the definition of the generalized TV seminorm in (2.17), the optimization problem is then given by

$$
\sup _{h \in C_{h}} \min _{f \in C_{f}}\left\{\left\|f-f_{0}\right\|_{2}^{2}+2 \lambda\left\langle f, L^{*} h\right\rangle\right\}
$$

where the adjoint $\mathrm{L}^{*}$ is

$$
\mathrm{L}^{*}=(-1)^{K} a_{K} \mathrm{D}^{K}+(-1)^{K-1} a_{K-1} \mathrm{D}^{K-1}+\cdots-a_{1} \mathrm{D}+a_{0} .
$$

The inner minimization problem can be solved by

$$
\tilde{f}=P_{C_{f}}\left(f_{0}-\lambda \mathrm{L}^{*} h\right),
$$

where $P_{C_{f}}$ is the projection onto $C_{f}=B_{l, u}$. If we substitute $\tilde{f}$ into (2.24), then (2.24) is reformulated as [2]

$$
\min _{h \in C_{h}}\left\{-\left\|P_{C_{f}}\left(f_{0}-\lambda \mathrm{L}^{*} h\right)-\left(f_{0}-\lambda \mathrm{L}^{*} h\right)\right\|_{2}^{2}+\left\|f_{0}(x)-\lambda \mathrm{L}^{*} h(x)\right\|_{2}^{2}\right\}
$$

and considering the objective function of $(2.27)$ as $q(h)$, the gradient of $q(h)$ is

$$
\nabla q(h)=2 \lambda \mathrm{L} P_{C_{f}}\left(f_{0}-\lambda \mathrm{L}^{*} h\right)
$$

Then, the optimal solution $\tilde{h}$ can be obtained by the gradient projection method

$$
h_{n}=P_{C_{h}}\left(h_{n-1}-\frac{2}{L(q)} \lambda \mathrm{L} P_{C_{f}}\left(f_{0}-\lambda \mathrm{L}^{*} h_{n-1}\right)\right)
$$

when $P_{C_{h}}$ denotes the projection on the convex set $C_{h}$, and $L(q)$ denotes the Lipschitz constant of $\nabla q(h)$. For the superlinear convergence rate, we implement the algorithm based on the Nesterov method $[2,21]$ as described below in Algorithm 1. Here, $L(q)$ denotes the Lipschitz constant of $\nabla q(h)$, which is upper-bounded by $16 \lambda^{2}[2]$, and $N$ is the number of the iteration.

Copyright $\odot$ by SIAM. Unauthorized reproduction of this article is prohibited. 


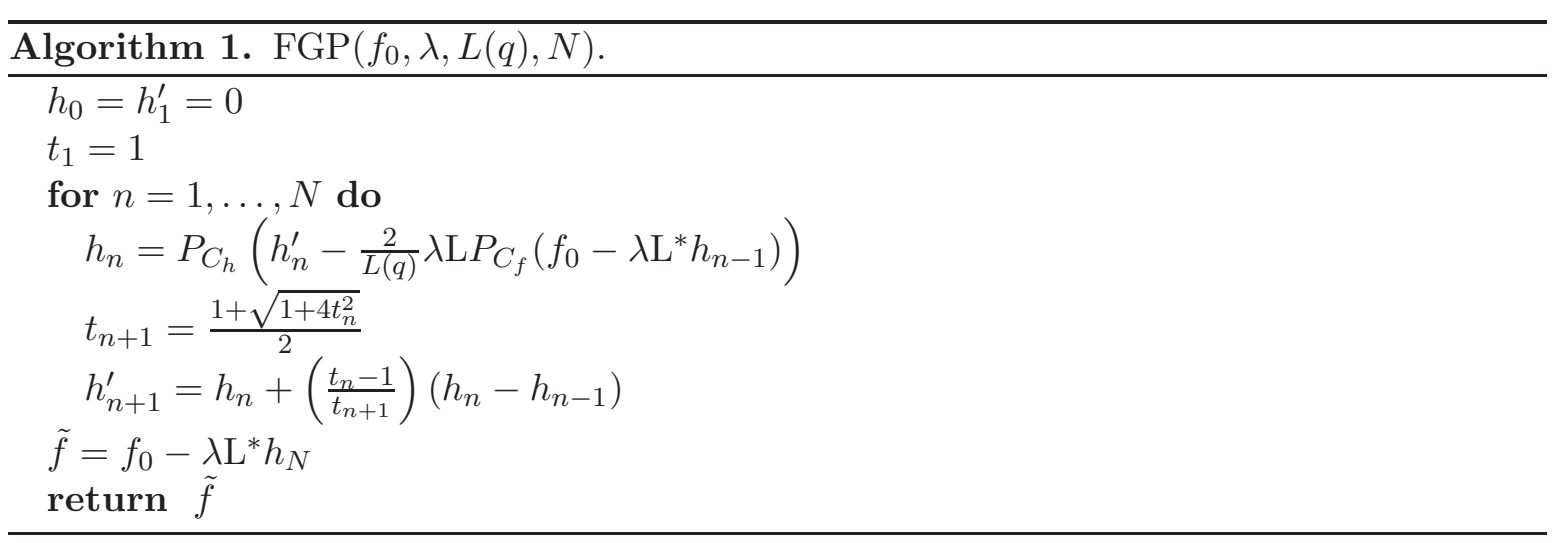

\section{Main contribution.}

3.1. Multiscale decomposition using the Bedrosian identity. The interior tomography using a 1D formulation described so far can make the optimization problem much simpler than the interior tomography using high-dimensional TV $[13,34,35]$. This was the main implementation approach taken in our previous work [32]. However, the method was still computationally expensive for practical use.

To accelerate the reconstruction speed to a level for clinical use, in this section, we propose a multiscale decomposition method by exploiting the powerful Bedrosian identity in the Hilbert transform. This idea is novel and was not addressed in our previous work [32]. More specifically, we assume that the object $f(x)$ is decomposed as

$$
f(x)=f_{L}(x)+f_{H}(x),
$$

where $f_{L}$ and $f_{H}$ denote the appropriate low and high frequency components of $f$, respectively, and $f_{L}$ is well modeled as an L-spline for some Fourier multiplier operator L. Under this assumption, our multiscale interior tomography problem is formulated as follows:

$$
\min _{f_{L}}\left\|f_{L}\right\|_{T V(L ; E)} \quad \text { subject to } \quad g=\mathcal{H}\left(f_{L}+f_{H}\right) \text {. }
$$

It is important to note that (3.1) is more realistic than (2.18) because images usually have textures or details that are often difficult to capture by a generalized TV. Moreover, even if the texture can be modeled as a higher order TV, the formulation in (3.1) still enables us to use a computationally more efficient lower order TV, as long as the remaining high frequency components can be reconstructed separately. In fact, such separate reconstruction of a high frequency component can be done accurately thanks to the Bedrosian theorem of the Hilbert transform.

Theorem 3.1 (see [19]). Let $w, f \in L_{2}(\mathbb{R})$ be low-pass and high-pass signals such that the Fourier transform of $w(x)$ vanishes for $|\omega|>\omega_{0}$, with $\omega_{0}>0$, and the Fourier transform of $f(x)$ vanishes for $|\omega|<\omega_{0}$. Then, we have

$$
\mathcal{H}\{w(x) f(x)\}=w(x) \mathcal{H}\{f(x)\}
$$

Copyright (c) by SIAM. Unauthorized reproduction of this article is prohibited. 
In order to appreciate the role of the Bedrosian theorem for the truncated Hilbert transform problem, note that the truncated DBP data can be represented as the product of the indicator function $w(x)$ that represents the truncation support and the untruncated DBP data $f(x)$ that corresponds to the Hilbert transform of the unknown signal. Then, (3.2) informs us that we can analytically recover the high frequency component $f_{H}$ which does not overlap with the spectrum of $w(x)$.

More specifically, after reconstructing $f_{L}(x)$ by solving (3.1), the residual signal $g_{H}(x)=$ $g(x)-\mathcal{H} f_{L}(x)$ can be obtained, which is equal to $\mathcal{H} f_{H}(x)$. Because we know $g_{H}(x)$ only in $E=\left(e_{1}, e_{2}\right)$, this can be considered as a multiplication of the rectangular window $w(x)=$ $\left[e_{1}, e_{2}\right]$ corresponding to the indicator function of the ROI whose spectral band is usually at low frequency ranges. If the spectral support of $g_{H}(x)$ does not overlap with that of $w(x)$, according to the Bedrosian theorem, we have

$$
w(x) g_{H}(x)=w(x) \mathcal{H} f_{H}(x)=\mathcal{H}\left\{w(x) f_{H}(x)\right\} .
$$

From (3.3), $f_{H}(x)$ can be analytically solved by

$$
f_{H}(x)=\frac{-\mathcal{H}\left\{w(x) g_{H}(x)\right\}}{w(x)}, \quad x \in E,
$$

which corresponds to applying the inverse Hilbert transform to the truncated residual DBP data and dividing the result by the window.

Figure 4 shows a step-by-step illustration of the multiscale decomposition method on a chord line. First, Figure 4(a) shows the low resolution signal $f_{L}(x)$ calculated by solving (3.1). Figure 4(b) shows the Hilbert transform of $f_{L}(x)$, whereas the gray dotted line is the original DBP data. Now, we compute the high-frequency component $g_{H}$ of the DBP data as the residual signal between the original DBP data and $\mathcal{H} f_{L}(x)$. Then, by taking the inverse Hilbert transform of $g_{H}(x)$, we can obtain the high frequency signal $f_{H}(x)$ shown in Figure 4(c). By summing up the signals in Figures 4(a) and (c), the final full-resolution reconstruction is obtained in Figure 4(d).

Note that (3.1) needs to be solved using an iterative method, and we use the POCS algorithm. However, the POCS reconstruction method using TV constraints often exhibits slow convergence in recovering high frequency signals. Interestingly, the multiscale decomposition from the Bedrosian identity also provides a remedy for this because an iterative approach can be used to recover only the low frequency component $f_{L}(x)$, and the iterative recovery of $f_{L}$ can be further accelerated by downsampling. More specifically, for a given ROI window $w(x)$ with the bandwidth of $\omega_{0}$, the measured DBP data are low-pass filtered with the bandwidth $\omega_{0}$ to obtain $g_{L}$ (see Figure 5). Because the signal content of $g_{L}$ is now low-passed, $g_{L}$ can be downsampled without aliasing at an appropriate downsampling factor. Then, POCS is applied to obtain a reconstruction in a downsampled domain, which is then upsampled to the original resolution to obtain the low frequency reconstruction $f_{L}$. The downsampling and upsampling procedure can be easily implemented by wavelet decomposition and reconstruction. Specifically, we should use the matched wavelets to the generalized TV operator L in (2.15). For example, if we use the first order TV operator (which is the case in our numerical experiments), the matched wavelet is represented by the Haar wavelet. Note that the com-

Copyright $\odot$ by SIAM. Unauthorized reproduction of this article is prohibited. 


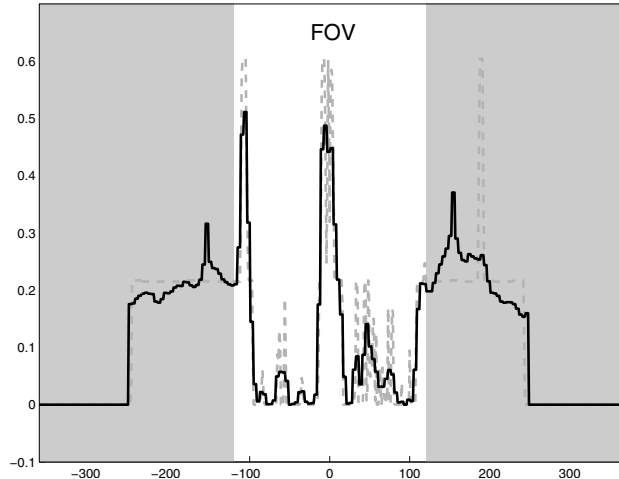

(a) $f_{L}(x)$

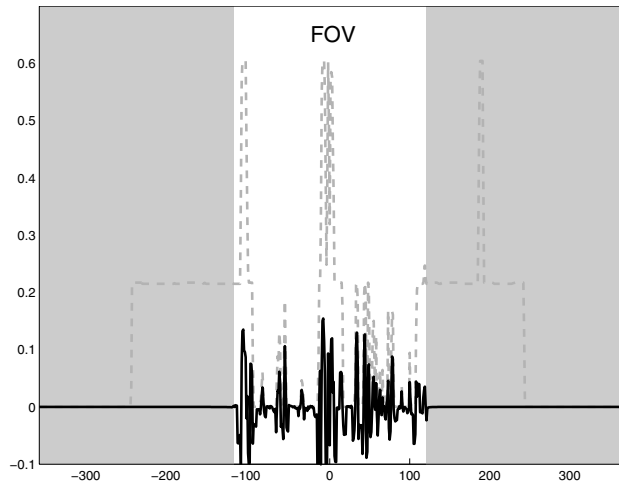

(c) $f_{H}(x)$

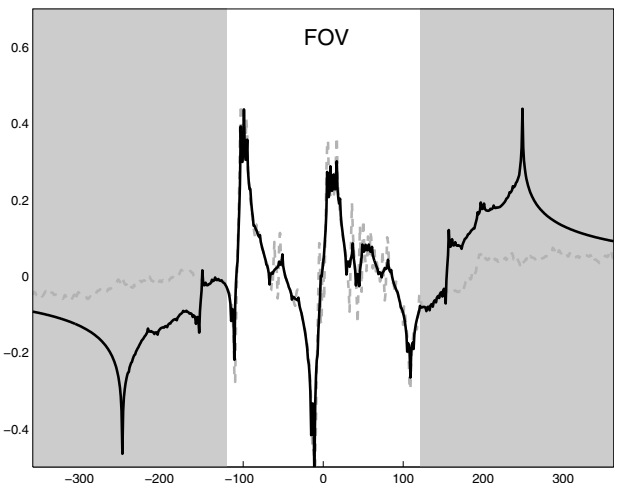

(b) $\mathcal{H} f_{L}(x)$

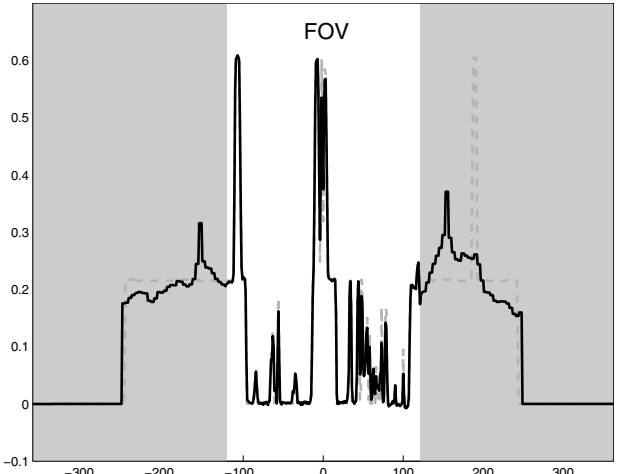

(d) $I(x)=f_{L}(x)+f_{H}(x)$

Figure 4. Step-by-step reconstruction procedure along a PI line.

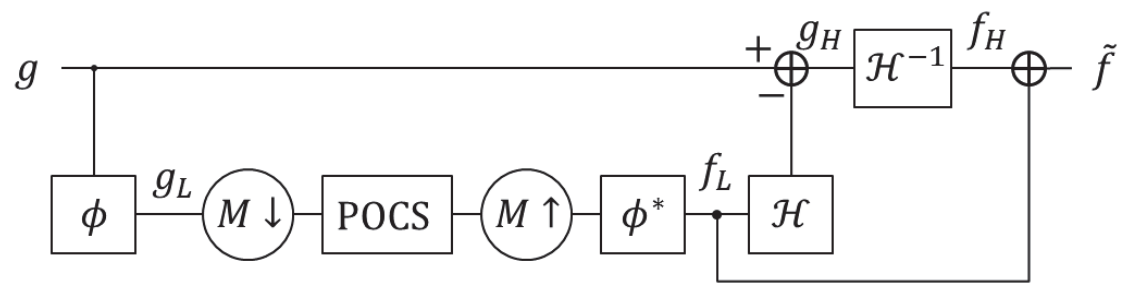

Figure 5. Flowchart of the proposed multiscale reconstruction approach for the interior tomography problem.

putationally expensive POCS operation is implemented in a downsampled domain, which is computationally much more efficient and provides faster convergence.

3.2. Spectral blending for minimizing the missing frequency. Having shown that the truncated Hilbert transform can be solved using a multiscale framework because of the Bedrosian theorem, the aim in this section is to show that a multiscale framework is feasible even for incomplete trajectories. This can be done by our spectral blending technique that minimizes the difference of $\phi(\cdot)$ in $(2.13)$ and $f(\cdot)$ in $(2.5)$. This part was inspired from the Fourier blending idea in [25] for half-scan imaging, but the detailed mathematical derivation 


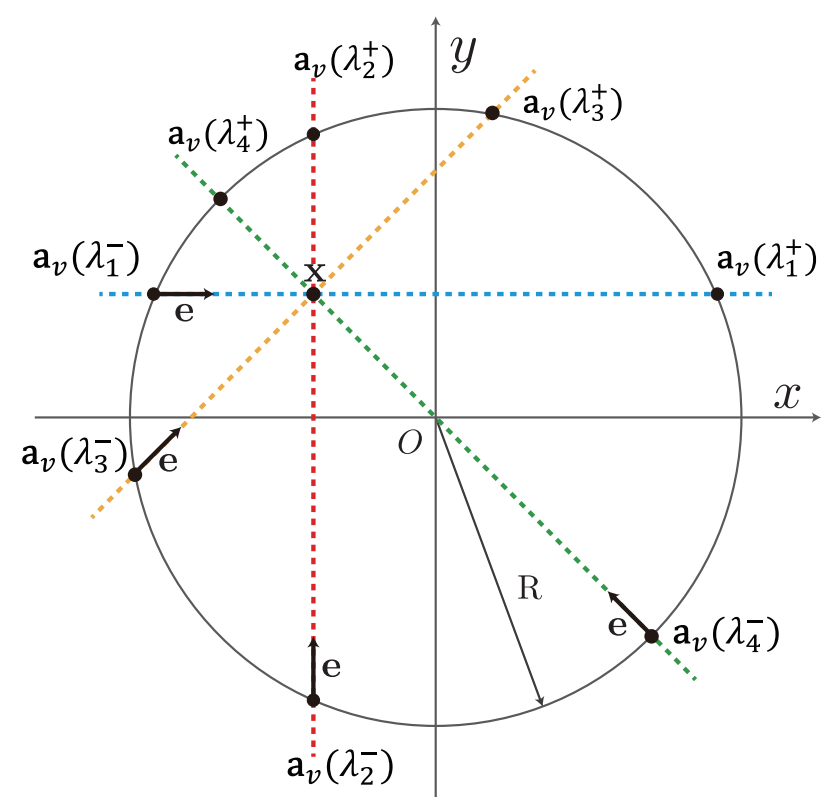

(a)

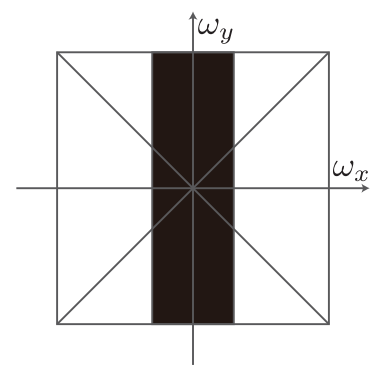

(b)

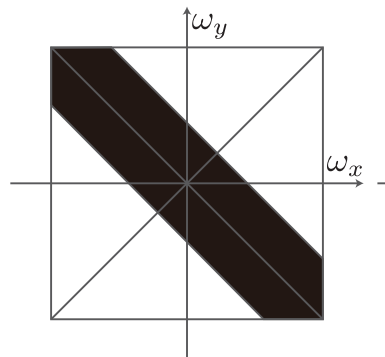

(d)

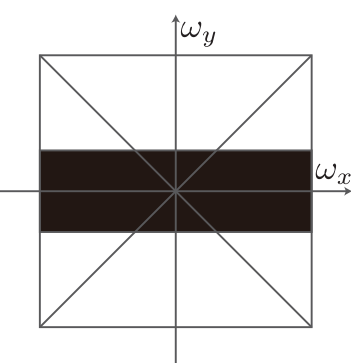

(c)

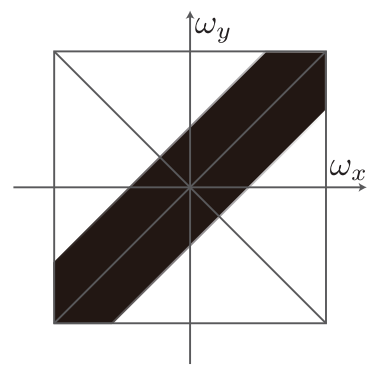

(e)

Figure 6. The missing frequency region. (a) The top view of a point $\mathbf{x}=(x, y, z)$ and a source trajectory. The blue line indicates the horizontal directional virtual chord line between $\mathbf{a}_{v}\left(\lambda_{1}^{-}\right)$and $\mathbf{a}_{v}\left(\lambda_{1}^{+}\right)$, and the red line denotes the vertical directional virtual chord lines between $\mathbf{a}_{v}\left(\lambda_{2}^{-}\right)$and $\mathbf{a}_{v}\left(\lambda_{2}^{+}\right)$. (b)-(c) The missing frequency region for the horizontal and vertical directions, respectively. (d)-(e) The missing frequency region for the diagonal directional virtual chord lines that correspond to the yellow and green lines, respectively.

is novel.

Accordingly with Theorem 2.2, the missing frequency region in the missing frequency set depends on the filtering direction. However, Theorem 2.2 also implies that at any point $\mathbf{x}$, if the missing frequency regions for different filtering directions are appropriately combined, we can minimize the artifacts from the missing frequency regions. This section describes this in detail.

Suppose that we are interested in recovering a voxel at $\mathbf{x}$ in Figure 6(a) from the DBP data from the actual source locations $\mathbf{a}(\lambda), \lambda \in\left[\lambda_{1}^{+}, \lambda_{1}^{-}\right]$. In Figure $6(\mathrm{a}), \mathbf{a}_{v}(\lambda)$ denotes the corresponding virtual source location. Then, (2.12) informs us that the missing frequency region for a given $z$ is given by

$$
\left\{\left(\omega_{x}, \omega_{y}, \omega_{z}\right) \mid-\frac{z \omega_{z}}{x+\sqrt{R^{2}-y^{2}}} \leq \omega_{x} \leq \frac{z \omega_{z}}{\sqrt{R^{2}-y^{2}}-x}\right\},
$$

where $z \omega_{z} \geq 0$; otherwise, the missing frequency region is given by

$$
\left\{\left(\omega_{x}, \omega_{y}, \omega_{z}\right) \mid \frac{z \omega_{z}}{\sqrt{R^{2}-y^{2}}-x} \leq \omega_{x} \leq-\frac{z \omega_{z}}{x+\sqrt{R^{2}-y^{2}}}\right\} .
$$

Note that the missing frequency region for a given slice at $z$ is also dependent on $x$ and $y$. However, if $z$ is close to the midplane (i.e., $z \simeq 0$ ), the missing frequency band vanishes. Thus, 


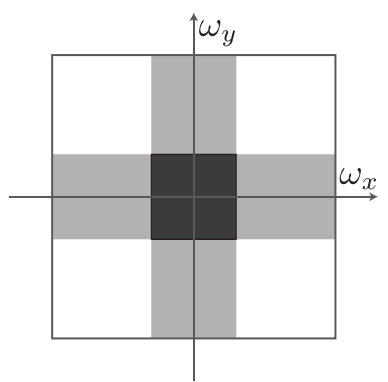

(a)

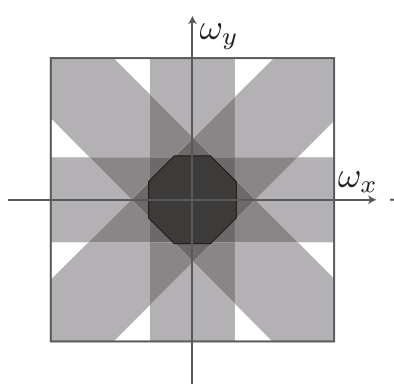

(b)

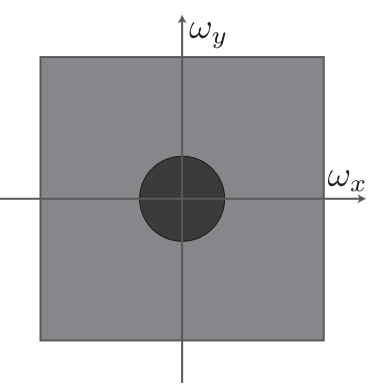

(c)

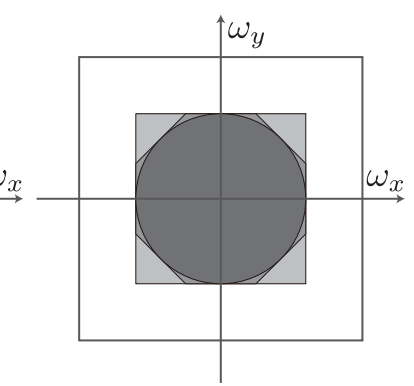

(d)

Figure 7. The common missing frequency region according to the filtering directions. (a), (b), and (c) indicate the missing frequency region according to the number of missing frequency regions with different filtering directions. The dark region denotes a common missing frequency region, respectively. (d) Overlapped illustration of the common missing frequency regions.

we are interested in slices far from the midplane, i.e., $x \ll z, y \ll z$. In this case, we have

$$
\left\{\left(\omega_{x}, \omega_{y}, \omega_{z}\right)|| \omega_{x} \mid \leq \frac{\left|z \omega_{z}\right|}{R}\right\}
$$

The resulting missing frequency region is illustrated in Figure 6(b). Similarly, the missing frequency region can be calculated for the vertical chord line direction between $\mathbf{a}_{v}\left(\lambda_{2}^{-}\right)$and $\mathbf{a}_{v}\left(\lambda_{2}^{+}\right)$, and as shown in Figure 6(c), the corresponding missing frequency region can be written as

$$
\left\{\left(\omega_{x}, \omega_{y}, \omega_{z}\right)|| \omega_{y} \mid \leq \frac{\left|z \omega_{z}\right|}{R}\right\}
$$

For diagonal directions along the yellow and green lines in Figure 6(a), the corresponding missing frequency region is described in Figures 6(d)-(e), respectively.

In Figures 7(a)-(c), the missing frequency regions from two, four, and infinitely many filtering directions are combined. Specifically, Figure $7(\mathrm{a})$ is constructed by combining the missing frequency regions from the two filtering directions, along the horizontal and vertical directions. In this case, the common missing frequency region is a dark square centered at the origin. In Figure 7(b), the frequency content from four directions (the horizontal, vertical, and two diagonal filtering directions) is combined. The common missing frequency region then corresponds to the dark hexagon centered at the origin, and the area of the common region is smaller than the missing region for the two directions in Figure 7(a). The minimum size of the common missing frequency region can be obtained by combining all angular filtering directions, and the resulting missing region should be circular, as shown in Figure 7(c). When the common missing frequency regions for Figures $7(\mathrm{a})-(\mathrm{c})$ are overlapped, all the common regions are included in the square obtained by the two-direction common missing frequency region shown in Figure 7(d). Note that the existence of the common missing frequency regions implies that the information within the common frequency cannot be recovered. However, except for the common frequency regions, if we have freedom to use the frequency contents of the reconstructions from the multiple filter directions, there is hope to recover the missing signal contents, which would not be possible using just a single filter direction.

Copyright (C) by SIAM. Unauthorized reproduction of this article is prohibited. 


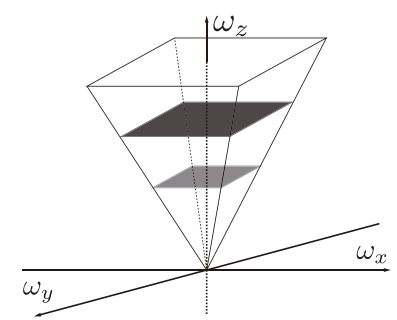

(a)

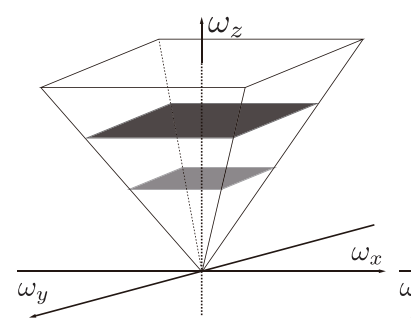

(d)

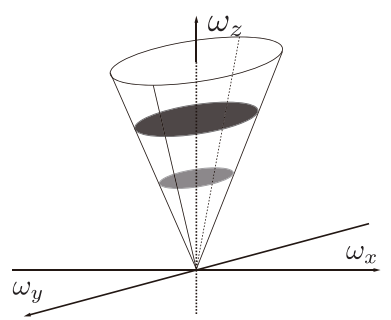

(b)

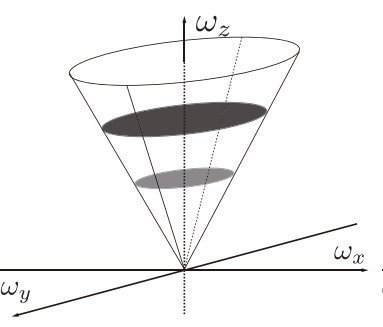

(e)

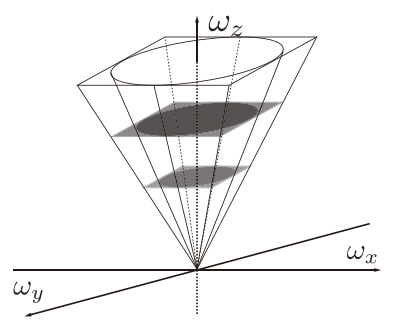

(c)

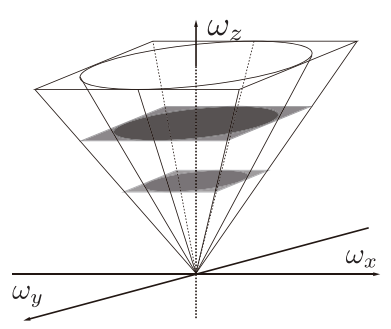

(f)

Figure 8. $3 D$ common missing frequency regions at two $\mathbf{x}$ 's with different $z$ 's. The first and second columns represent the common missing frequency regions for the 2-direction and infinite filtering direction, respectively. The last column illustrates the overlaps of the two missing frequency regions. The $z_{2}$ corresponding to the second row is further from the midplane than $z_{1}$ of the first row.

Specifically, consider the quadrangular pyramids and cones in Figures 8(a)-(d) (and Figures $8(\mathrm{~b})-(\mathrm{e}))$ that correspond to the $3 \mathrm{D}$ common missing frequency regions at two different $z$ locations when two (resp., multiple) filtering directions are combined. Note that the missing frequency regions have different geometries depending on the positions of $z$. Specifically, as $z$ is placed far away from the midplane, the bigger the missing frequency region becomes, as shown in Figure 8. Fortunately, the shapes of the missing frequency regions are still the same regardless of the $z$ positions, even though their sizes are different. This leads to the spatially invariant spectral blending scheme that results in the minimized missing frequency regions. More specifically, for the case of spectral blending using two orthogonal filtering directions, we may want to use the frequency region corresponding to the shaded area of the bow tie in Figure 9(a) for the reconstruction images from the horizontal filtering line. Similarly, we can use the bow tie in Figure 9(b) for reconstructing images from the vertical filtering line. Because the shape of the missing region remains the same, it is possible to blend the missing frequency region like Figure 9(c) by using a 2D Fourier transform at each slice. Then, the resulting missing frequency region becomes equal to Figures $8(\mathrm{a})-(\mathrm{d})$, which is the minimum size missing frequency region we can expect from the two distinct filtering directions.

The step-by-step procedure of spectral blending can simply be expressed as Algorithm 2 and is shown in Figure 10. Our multiscale interior tomography algorithm is used for both horizontal and vertical directional reconstructions, and they are blended into one image using spectral weighting with the bow tie window.

4. Experimental results. The proposed method was verified using numerical experiments. We implemented the proposed multiscale interior tomography algorithm for 2D fan-beam CT, 


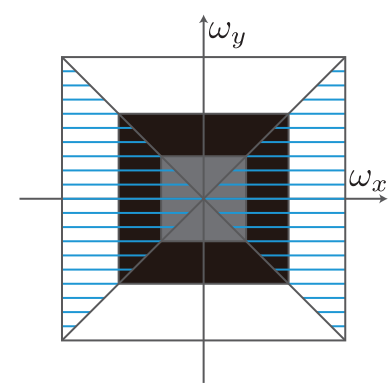

(a)

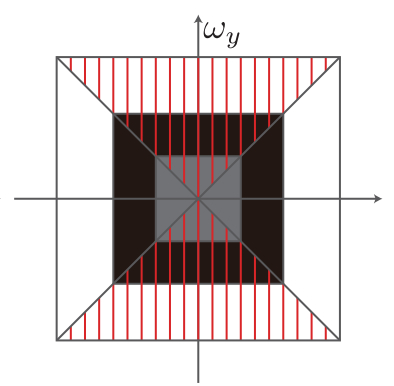

(b)

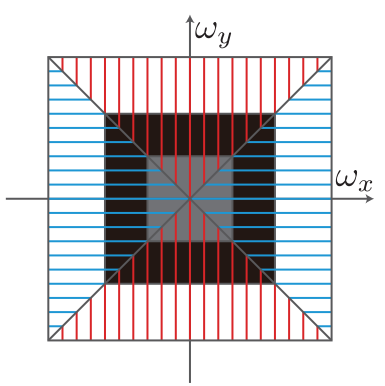

(c)

Figure 9. Spectral weighting function for two orthogonal filtering directions. (a)-(b) Spectral weighting for the image reconstructed using horizontal and vertical chord lines, respectively. The spectral components in the shaded areas are used. (c) Composite spectral weighting.

Algorithm 2. Spectral blending.

1: Reconstruct the objects $\phi(x)$ and $\phi^{\perp}(x)$ with the orthogonal direction of the chord line, respectively.

2: For each slice, perform the 2D Fourier transform

$$
\hat{\phi}(\omega)=\mathcal{F}\{\phi(x)\}, \hat{\phi}^{\perp}(\omega)=\mathcal{F}\left\{\phi^{\perp}(x)\right\} .
$$

3: Reconstruct a final object $\tilde{f}(x)$ by performing the spectral blending such that

$$
\tilde{f}(x)=\mathcal{F}^{-1}\left(W \hat{\phi}(\omega)+(1-W) \hat{\phi}^{\perp}(\omega)\right) .
$$

3D helical cone-beam CT, and 3D circular cone-beam CT. To demonstrate that the algorithm is very practical for clinical applications, all the numerical experiments were performed using typical clinical CT geometry parameter settings.

In the POCS algorithm, we need the boundary of an object support, but the exact boundary is hardly known in real scanning cases. Thus, for a realistic simulation, we assumed that only the maximum width and height of the body $\left(W_{b o d y}, H_{b o d y}\right)$ in the transverse planes are known, and then the approximate boundary is defined as an ellipse with a bigger width and height than that of $W_{b o d y}$ and $H_{b o d y}$. The width and height of the ellipse are indicated as $W_{\text {ellipse }}$ and $H_{\text {ellipse }}$ in

$$
\begin{aligned}
& W_{\text {ellipse }}=W_{\text {body }}+L_{\text {extension }}, \\
& H_{\text {ellipse }}=H_{\text {body }}+L_{\text {extension }}
\end{aligned}
$$

with the positive real number $L_{\text {extension. }}$. As in Figure 11, we can define ellipses with various $L_{\text {extension. }}$ In some cases, the ellipse could not cover the whole support, such as the arms. In the reconstruction simulation of a circular fan-beam and helical cone-beam CT, we used the ellipse boundary when $L_{\text {extension }}$ was $60 \mathrm{~mm}$.

4.1. 2D fan-beam CT. The first reconstruction result was obtained from a $2 \mathrm{D}$ circular fan-beam projection, in which the projection data at each view were truncated. To cover the 

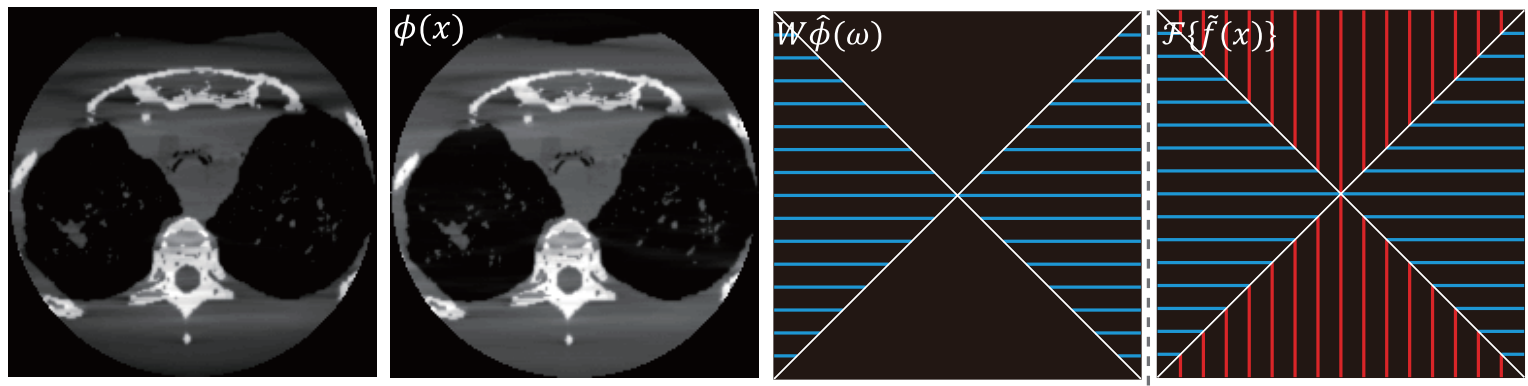

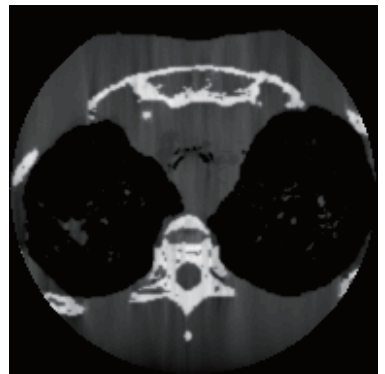

(a)

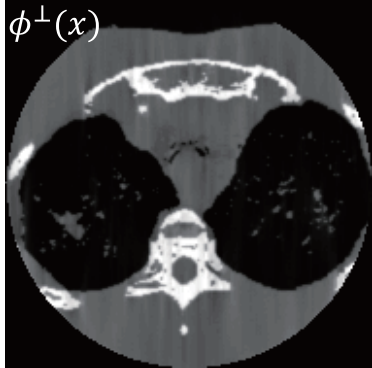

(b)

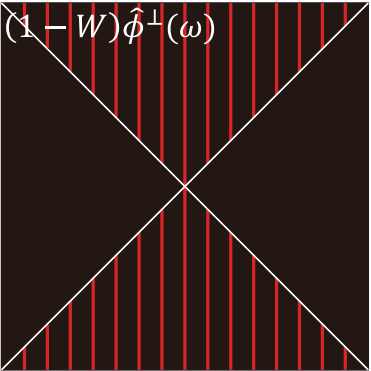

(c)

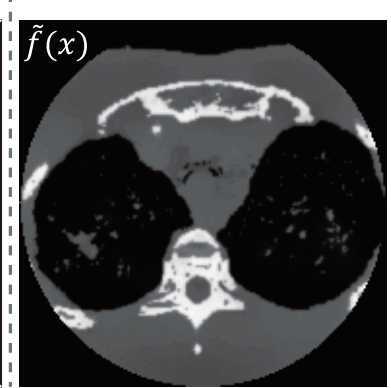

c)

Figure 10. ROI tomography reconstruction results using (a) BPF, (b) the proposed multiscale reconstruction method along the horizontal $(\phi(x))$ and vertical $\left(\phi^{\perp}(x)\right)$ filter directions, and (c) the spectral blending results from the two filtering directions.

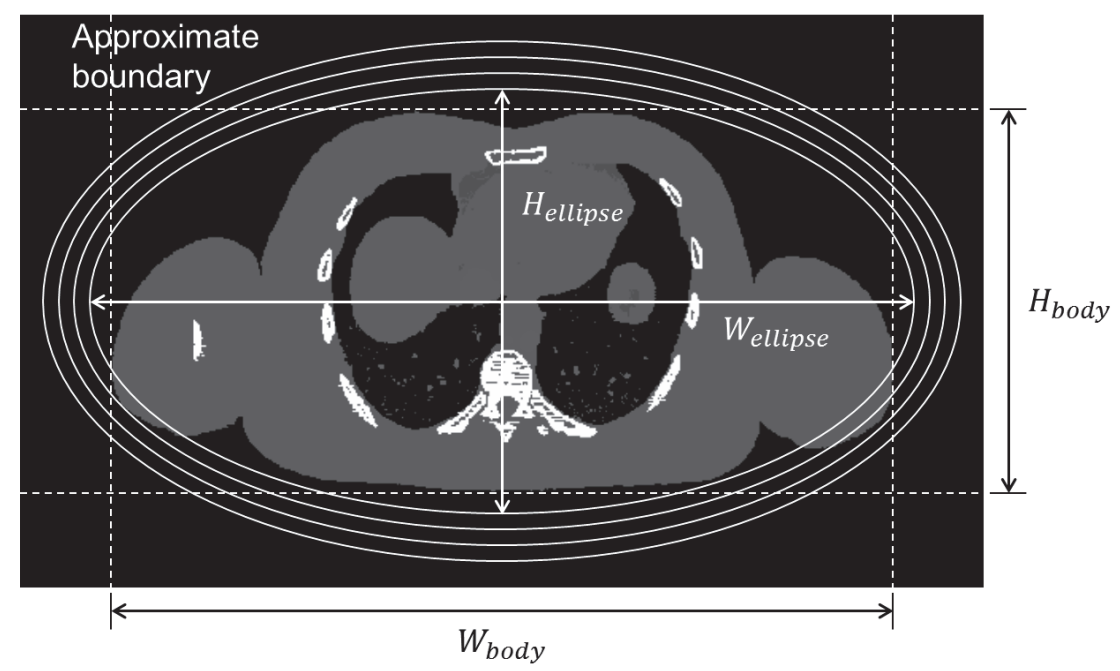

Figure 11. Approximate ellipse boundary.

whole ROI area, we chose a set of filtering lines or chord lines along the vertical direction. The sampling distance on the chord line is the same as the reconstruction grid size. Even if the chord line covers the whole object, the DBP data are necessary only for the ROI because the DBP outside the ROI cannot be computed correctly due to detector truncation. For the

Copyright (c) by SIAM. Unauthorized reproduction of this article is prohibited. 


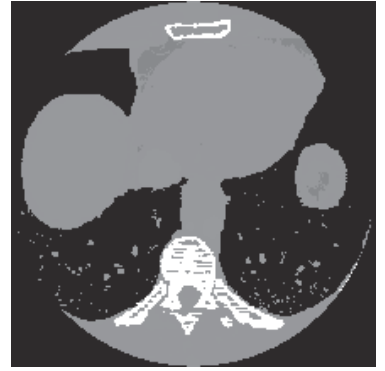

(a) Ground truth

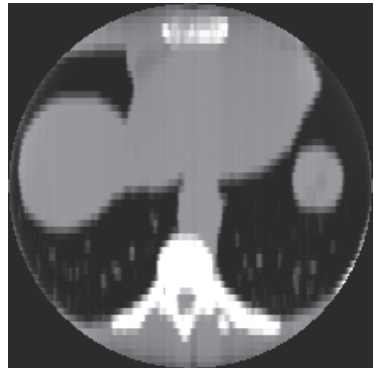

(b) $f_{L}$

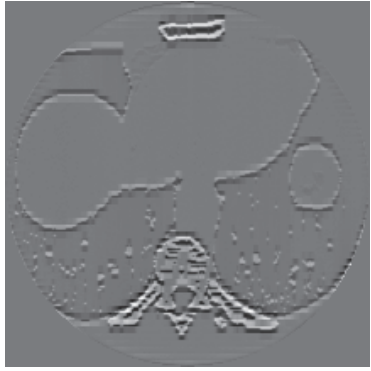

(c) $f_{H}$

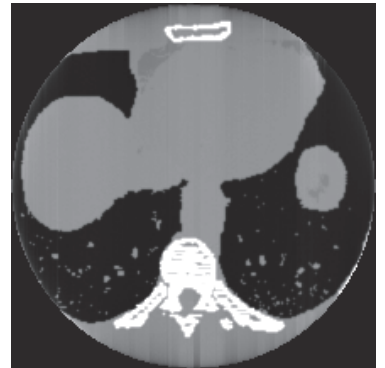

(d) $f_{L}+f_{H}$

Figure 12. $2 D$ fan-beam reconstruction. For the simulation, the $K$ of the $\mathrm{L}$ is 1 , and the iteration number of the POCS is 100. The $l$ and $u$ are 0 and 0.060442 , respectively, and the $\lambda$ is 0.001 , and $N$ is 20. The depth of the multiscale implementation is 2. (a) Ground truth, (b) low frequency signal reconstruction, (c) high frequency signal reconstruction, and (d) the final reconstruction result.

simulation, we used a 2D inner organ phantom, as shown in Figure 11, which is $512 \times 512$ in size with a $1 \times 1 \mathrm{~mm}^{2}$ size pixel. The number of detectors in the array was 380 with a $1 \mathrm{~mm}$ pitch, and the number of views was 1200 . The distance from the source to the rotation axis was 800 $\mathrm{mm}$, and the distance from the source to the detector was $1400 \mathrm{~mm}$. The radius of the ROI was about $107 \mathrm{~mm}$, which cannot cover the whole object. For the multiscale implementation, we used the first order operator L in (2.15), which corresponds to the conventional TV penalty. The iteration number of the POCS was 100. For Algorithm 1, the $l$ and $u$ in (2.23) were 0 and 0.060442 , respectively, for the inner organ phantom, and the $\lambda$ was 0.001 , and $N$ was 20 . For the multiscale implementation, the depth of Haar wavelet decomposition was 2.

In Figure 12, the first image in (a) is the original ground truth. Figures 12(b)-(c) show the low and high frequency reconstruction images, respectively, and the final reconstruction is shown in Figure 12(d), which is nearly identical to the original image.

4.2. 3D helical cone-beam CT. The second experiment is from a 3D cone-beam projection with a helical scanning trajectory, where the projections at each view extend only to the shadow of a cylindrical region completely interior to the subject being scanned. This situation is important for low-dose cardiac CT imaging using a clinical multislice helical CT.

To cover the whole 3D ROI area, we defined the PI lines shown in Figure 13. First, $\lambda^{-}$was sampled along the helical trajectory such that the increment of $\mathbf{a}\left(\lambda^{-}\right)$along the $z$-axis should be shorter than the reconstruction grid size along the $z$-axis in the 3D Cartesian coordinate system. Second, $\lambda_{\min }^{+}$and $\lambda_{\max }^{+}$were chosen to cover only the ROI, and $\lambda^{+}$was sampled such that the increment of $\mathbf{a}\left(\lambda^{+}\right)$in the $x y$-plane should be smaller than the reconstruction grid size in the $x y$-plane. Third, the $\mathbf{x}$ along the PI line $\mathcal{L}\left(\lambda^{-}, \lambda^{+}\right)$that connects $\mathbf{a}\left(\lambda^{-}\right)$to $\mathbf{a}\left(\lambda^{+}\right)$ was sampled to cover the object support boundary $\left(b_{1}, b_{2}\right)$. The sampling distance on the PI line should be determined such that the corresponding projection on the $x y$-plane should be smaller than the reconstruction grid size in the $x y$-plane. To calculate the DBP data at $\mathbf{x}$ on $\mathcal{L}\left(\lambda^{-}, \lambda^{+}\right)$, backprojections are required for $\mathcal{C}\left(\lambda^{-}, \lambda^{+}\right)$, and the DBP data are necessary only for the ROI $\left(e_{1}, e_{2}\right)$.

For helical cone-beam simulation, we used two kinds of 3D phantoms: XCAT and inner organ phantoms. For comparison, we implemented two types of analytic exact cone-beam 


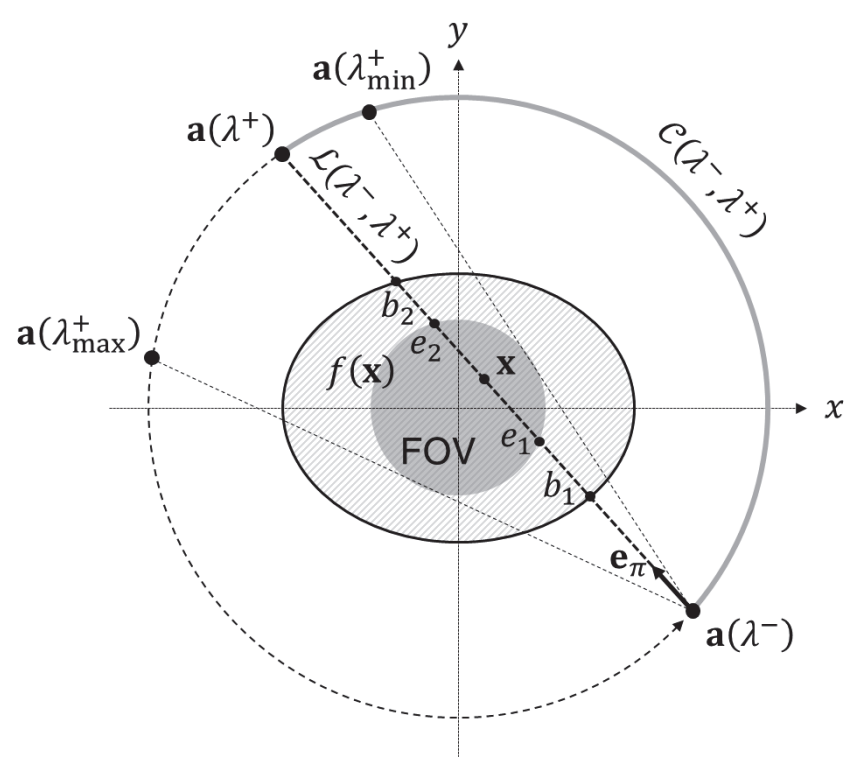

Figure 13. Implementation of helical trajectory.

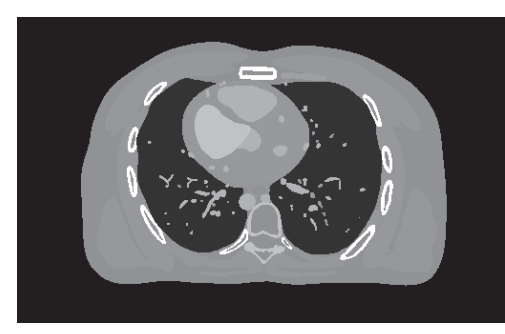

Transverse

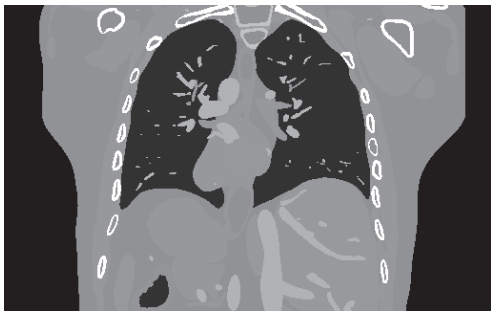

Coronal

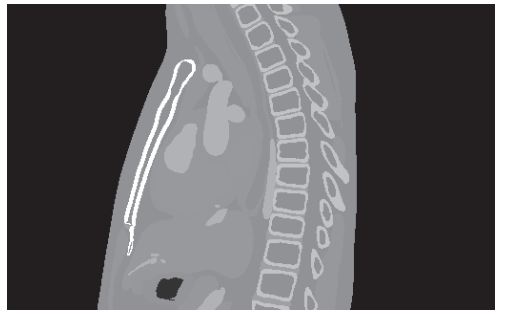

Sagittal

Figure 14. $3 D X C A T$ phantom.

reconstruction methods: the Katsevich-type FBP [16] and BPF algorithm, and the fully iterative reconstruction method using the $3 \mathrm{D}$ TV regularizer. The first result is for the XCAT phantom, and its resolution was $512 \times 512 \times 320$ voxels with a voxel size $1 \times 1 \times 1 \mathrm{~mm}^{3}$. Figure 14 shows the transverse, coronal, and sagittal planes of the phantom. The distances from the source to the rotation axis and from the source to the detector were the same as in the circular fan-beam simulation. The detector resolution was $400 \times 109$ pixels with a pitch of $1 \times 1 \mathrm{~mm}^{2}$; thus the radius of the ROI was about $127 \mathrm{~mm}$. For the helical scanning trajectory, the number of rotations was 3, the helical pitch $H$ was $100 \mathrm{~mm}$, and the number of views was 800 per rotation. The object was reconstructed in the PI line space first, and the resolution of the PI line space to cover the ROI was $727 \times 245 \times 339$. After reconstructing all the PI lines, the final reconstruction of about 236 slices was obtained by the regridding process. For the simulation parameters, the $K$ of the L, the iteration number of the POCS, $\lambda, N$, and the depth were all the same as the fan-beam simulation, and the $l$ and $u$ were 0 and 0.030888 , respectively.

Figure 15 shows the reconstruction result in the ROI by FBP, BPF, 3D TV, and the 

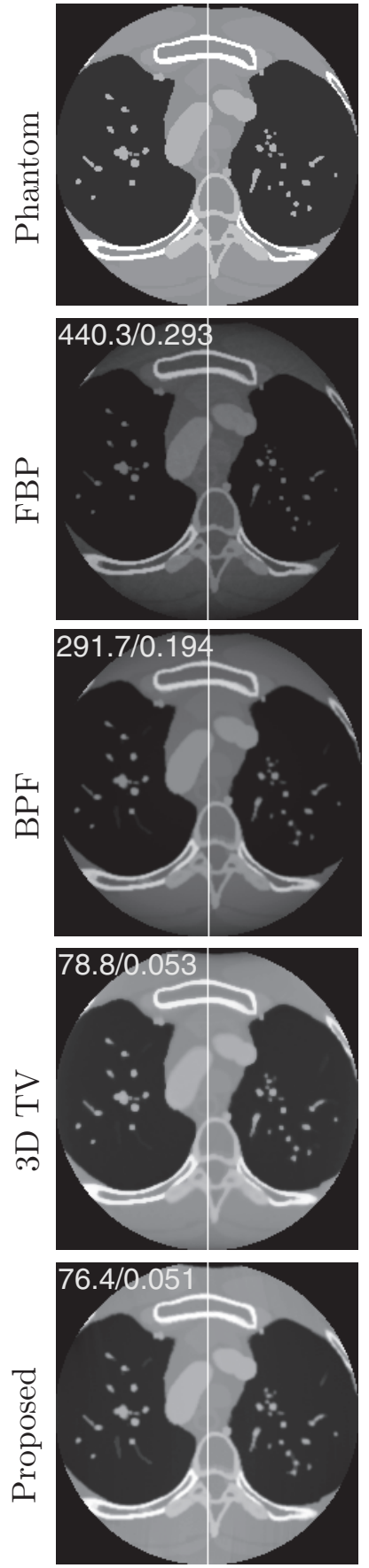

(a) 84
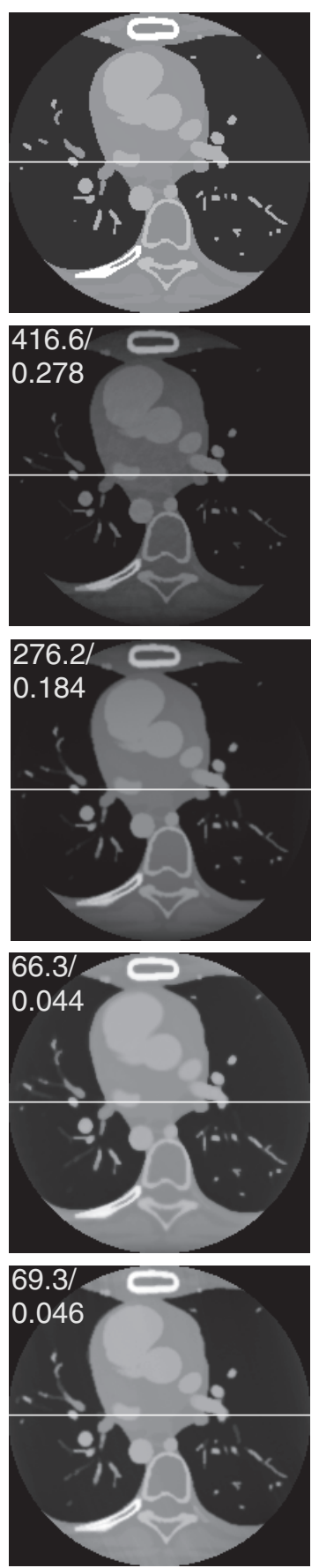

(b) 130
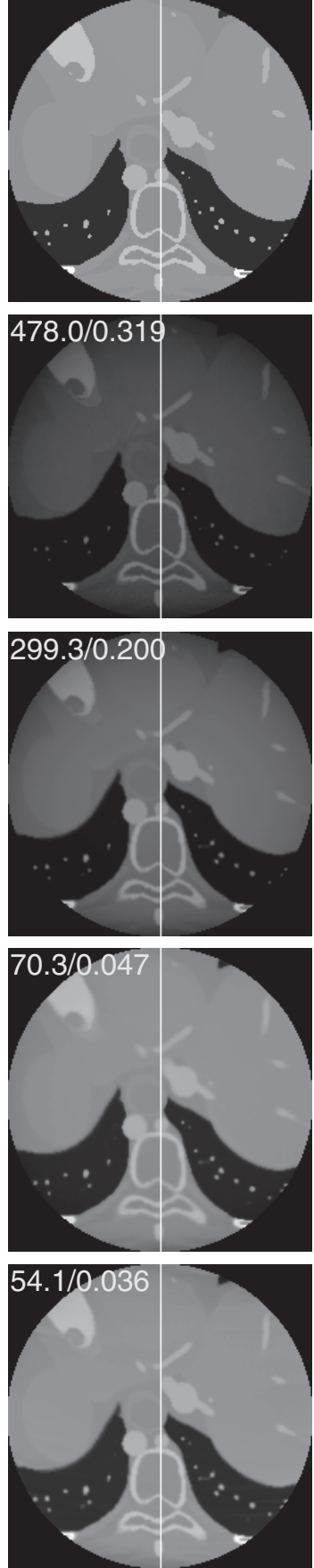

(c) 212

Figure 15. Reconstructed images by the truncated helical cone-beam simulation of the $3 D$ XCAT phantom. For the simulation, the $K$ of the $\mathrm{L}$ is 1 , and the iteration number of the POCS is 100 . The $l$ and $u$ are 0 and 0.0309 , respectively, and the $\lambda$ is 0.001 , and $N$ is 20. The depth of the multiscale implementation is 2. From the first to third columns, each column shows the slice image when the slice number is (a) 84, (b) 130, and (c) 212, and each row is for the ideal phantom, FBP, BPF, 3D TV, and the proposed method. The last column shows the cut views indicated by the white line on the images. The number written at the corner of the reconstructed image shows the RMSE and NRMSE in the Hounsfield unit (HU) scale.

Copyright (C) by SIAM. Unauthorized reproduction of this article is prohibited. 


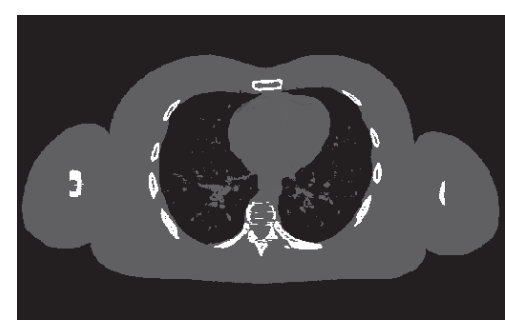

Transverse

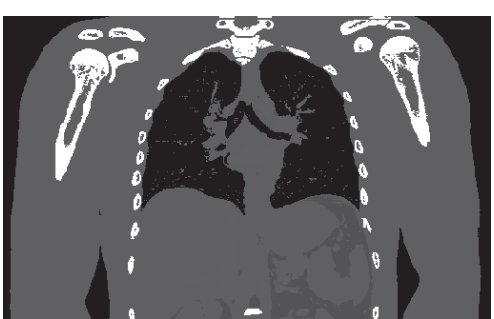

Coronal

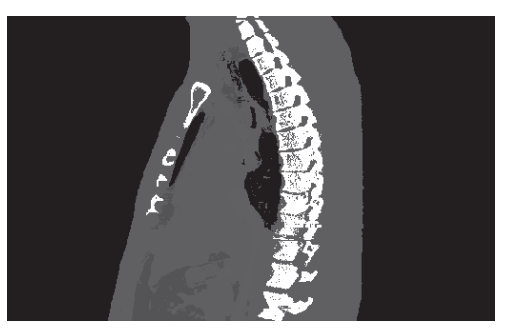

Sagittal

Figure 16. $3 D$ inner organ phantom.

proposed method. From the first to third columns, the first row is for the ideal phantom, and the second to fourth rows are for the reconstructed images by FBP, BPF, 3D TV, and the proposed method, respectively. As shown in the images of Figure 15, it is clearly observed that the FBP images are much darker compared to the original phantom, and the BPF images are still dark, while the images by our proposed method show almost the same intensity as the original. Even when comparing the result of the 3D TV method, the proposed method is competitive. This can also be seen in the cut view, which is the last column of Figure 15. Moreover, the root-mean-square error (RMSE) and normalized root-mean-square error (NRMSE) values indicated on the images provide clear quantitative evidence.

The second result is for the noisy simulation of the 3D inner organ phantom. Figure 16 shows the transverse, coronal, and sagittal planes of the phantom. To make the noisy projection data, we assumed the detector measurements have a Poisson distribution, and the signal-to-noise ratio (SNR) was set to about $30 \mathrm{~dB}$ in the intensity domain. The helical CT geometry was the same as before, and the hyperparameters for the proposed interior tomography algorithm were the same as those for the 2D experiments.

As shown in Figure 17, the FBP and BPF methods still show dark images, and the 3D TV method and the proposed method recover the images correctly. However, there is a difference between the 3D TV and the proposed algorithms. Compared to the reconstruction without truncation, the recovered textures of the proposed algorithm are very similar, which indicates that the algorithm did not provide unnatural smoothings. On the other hand, the 3D TV algorithm reduces not only the noise of the image but also the details. The noise reduction is necessary for some cases, but it can also remove important structures of the image. Our proposed algorithm conserved the details of the image thanks to the analytic reconstruction of the high frequency details.

Tables 1 and 2 show the computational time of each process step in the case of the 3D inner organ phantom simulation. In Table 1 , time was measured by increasing the truncation rate (TR) with a depth of 2 , and the TR corresponds to

$$
\mathrm{TR}=\frac{l-l_{T}}{l} \times 100 \%,
$$

where $l$ and $l_{T}$ denote the length of the original and truncated detector, respectively. For reference, the result in Figure 17 is for the case in which the truncation rate was $60.9 \%$. All processes were implemented by a GPU to accelerate the reconstruction speed, and a whole

Copyright (C) by SIAM. Unauthorized reproduction of this article is prohibited. 

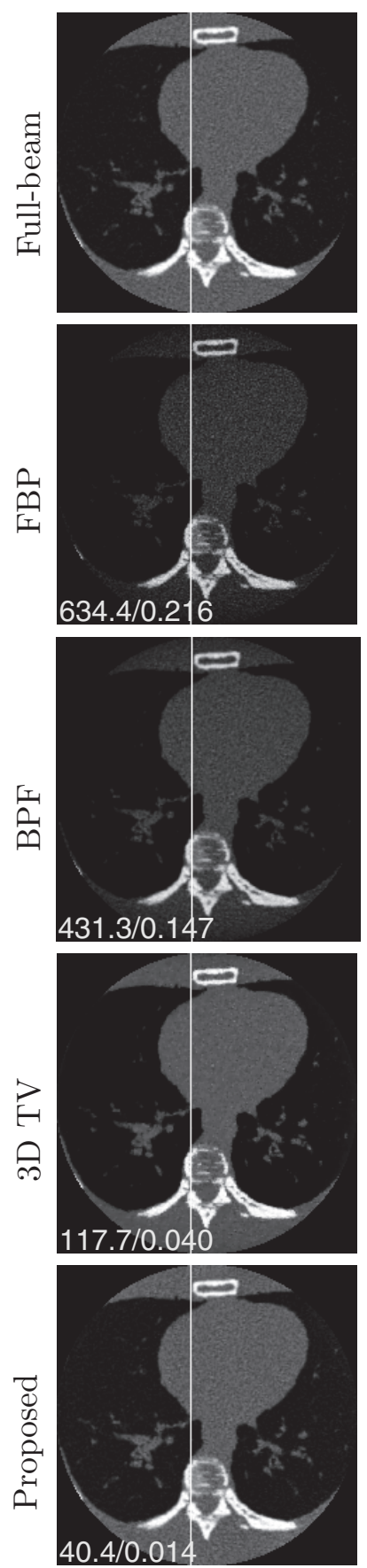

(a) Transverse
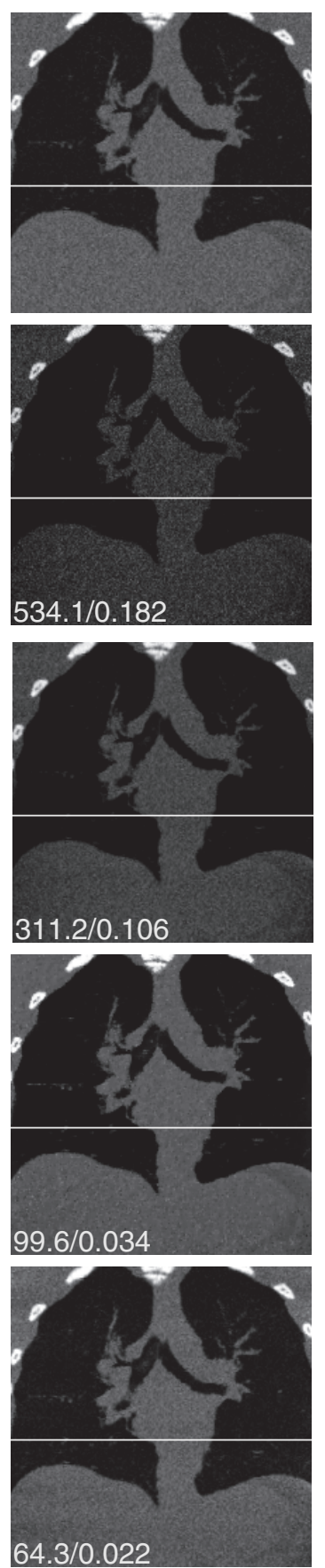

(b) Coronal
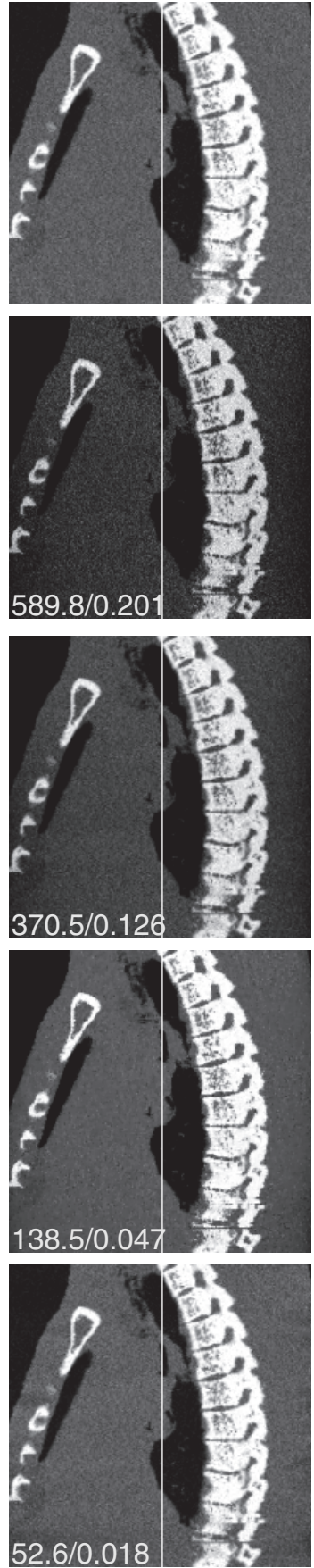

(c) Sagittal
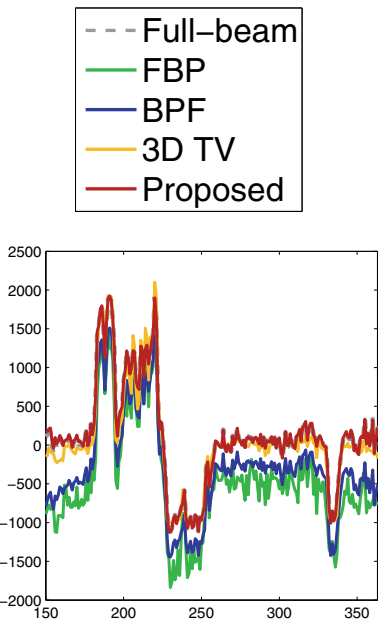

(a) Transverse

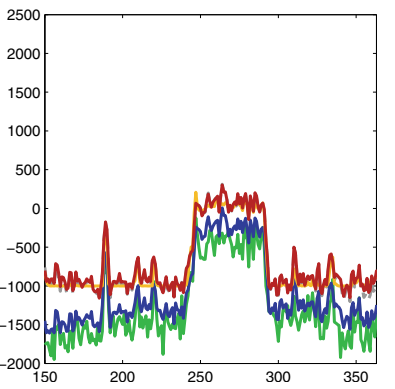

(b) Coronal

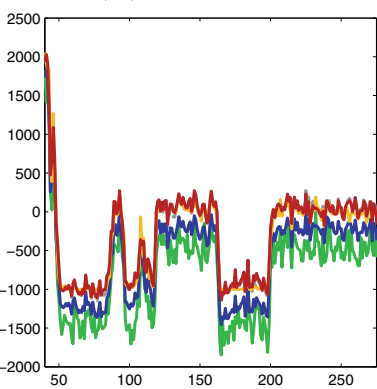

(c) Sagittal

Figure 17. Reconstructed images by the truncated helical cone-beam simulation of the $3 D$ inner organ phantom with noise. For the simulation, the $K$ of the $\mathrm{L}$ is 1 , and the iteration number of the POCS is 100. The $l$ and $u$ are 0 and 0.0604, respectively, and the $\lambda$ is 0.001, and $N$ is 20. The depth of the multiscale implementation is 2. From the first to third columns, each column shows the same (a) transverse, (b) coronal, and (c) sagittal image, and each row is for full-beam BPF, FBP, BPF, 3D TV, and the proposed method. The last column shows the cut views indicated by the white line on the images. The number written at the corner of the reconstructed image shows the RMSE and NRMSE in the HU scale. 
Table 1

Computational time (seconds) by truncation rate for helical cone-beam CT when depth is 2 .

\begin{tabular}{lrrr}
\hline & \multicolumn{3}{c}{ Proposed } \\
\cline { 2 - 4 } TR & $56.1 \%$ & $60.9 \%$ & $65.8 \%$ \\
\hline DBP & 14.1 & 13.7 & 13.3 \\
Downsampling & 0.7 & 0.6 & 0.5 \\
$f_{L}$ reconstruction & 85.4 & 170.4 & 335.1 \\
Upsampling & 0.3 & 0.3 & 0.2 \\
$f_{H}$ reconstruction & 9.1 & 8.9 & 8.6 \\
Regridding & 2.7 & 2.5 & 2.2 \\
\hline Total $(236$ slices) & 112.3 & 196.4 & 359.9 \\
Slices/sec & 2.102 & 1.202 & 0.656 \\
\hline
\end{tabular}

Table 2

Computational time (seconds) by depth for helical cone-beam CT when truncation rate is $60.9 \%$.

\begin{tabular}{lrrrr}
\hline & 3D TV & \multicolumn{3}{c}{ Proposed } \\
\cline { 2 - 5 } Depth & & 0 & 1 & 2 \\
\hline DBP & & 13.7 & 13.8 & 13.7 \\
Downsampling & 0 & 0.4 & 0.6 \\
$f_{L}$ reconstruction & 1418.5 & 558.5 & 170.4 \\
Upsampling & 0 & 0.1 & 0.3 \\
$f_{H}$ reconstruction & & 8.5 & 8.4 & 8.9 \\
Regridding & & 2.6 & 2.5 & 2.5 \\
\hline Total (236 slices) & 3141.2 & 1443.3 & 583.7 & 196.4 \\
Slices/sec & 0.075 & 0.164 & 0.404 & 1.202 \\
\hline
\end{tabular}

Table 3

Whole workstation environment for simulating the proposed method.

\begin{tabular}{cc}
\hline System & Specification \\
\hline CPU & Intel Core i7-4700 Haswell Quad-Core $3.40 \mathrm{GHz}$ \\
RAM & Samsung 16GB kit $(4 \times 4 \mathrm{~GB})$ DDR3 PC3-128000, 1600MHz \\
GPU & GeForce GTX TITAN \\
OS & Windows 7 Enterprise with CUDA 5.5 \\
\hline
\end{tabular}

workstation environment is presented in Table 3. Table 1 shows more computational burden with a more severe truncation due to the increase in the iteration number for convergence. Because the time for the low frequency reconstruction is the longest compared to all the others, the total amount of time mainly consists of the low frequency part.

On the other hand, Table 2 shows a reduced computational time with more multiresolution decomposition. Table 2 also shows the computational time of the $3 \mathrm{D}$ TV method. In Table 2, the computational time of the 3D TV method is quite long because the process for the 3D helical projection and backprojection is repeated. However, the computation time of the proposed method is an order of magnitude faster than that of the 3D TV method.

Copyright $\odot$ by SIAM. Unauthorized reproduction of this article is prohibited. 
Moreover, by increasing the depth, the time for the low frequency reconstruction rapidly decreases to a practical level. Table 2 confirms that the computational time is quite reasonable (1.2 slices/second) even for clinical multislice helical CT applications. This is because of the multiscale decomposition which leads to a dramatic reduction in the reconstruction time.

4.3. 3D circular cone-beam CT. For the circular cone-beam simulation, we also used two kinds of $3 \mathrm{D}$ phantoms which are the same as the helical cone-beam simulation. Again, the experimental scenario was for the classic interior problem, which is a limited data sampling problem where projections at each view extend only to the shadow of a cylindrical region completely interior to the subject being scanned. Moreover, due to the cone-beam artifact, there exist additional technical huddles in the image reconstruction.

For comparison, we implemented two types of analytic cone-beam reconstruction methods: the Feldkamp-Davis-Kress (FDK) and BPF algorithm, and the fully iterative reconstruction method using the 3D TV regularizer. The first result is for the XCAT phantom, which is equal to the previous one. The distances from the source to the rotation axis and from the source to the detector were the same as in the circular fan-beam simulation. The detector resolution was $350 \times 640$ pixels with a pitch of $1 \times 1 \mathrm{~mm}^{2}$; thus the radius of the ROI was about $94 \mathrm{~mm}$, and the number of views was 1000. The simulation parameters were the same as in the fan-beam and helical cone-beam cases, except for the iteration number of the POCS, and $\lambda$. In the 3D circular cone-beam CT case, the iteration number of the POCS was 30, and $\lambda$ was 0.01 and 0.02 for the inner organ phantom and XCAT phantom, respectively.

Figure 18 shows the reconstruction result in the ROI by FDK, BPF, 3D TV, and the proposed method. The first row is for the ideal phantom, and the second to fourth rows are for the reconstructed images by FDK, BPF, 3D TV, and the proposed method, respectively. Each column represents the 50th, 105th, and 180th slices when the midplane is the 160th slice. The reconstructed images are similar to the helical cone-beam simulation results, except that the FDK images are brighter than that of the original phantom. Figure 19 shows the noisy simulation of the 3D inner organ phantom. The artifact patterns from the existing reconstruction algorithms were also noticeable, whereas the details of the image are well conserved by the proposed method.

Table 4 compares the reconstruction time using the conventional 3D TV approach and the proposed method. All processes were implemented by a GPU to accelerate the reconstruction speed. The proposed method significantly outperformed the conventional approach by an order of magnitude acceleration, and it achieved a speed of three slices/second reconstruction.

\section{Discussion.}

5.1. Comparison with the extrapolation method. To demonstrate the limitation of the conventional method using sinogram extrapolation, we compared the conventional method and our proposed method by increasing the $L_{\text {extension }}$ in (4.1). In our POCS implementation, we used information from the approximate ellipse boundary, and thus in the extrapolation method, we also used the same approximate ellipse boundary to determine the extension length of the sinogram. The extrapolation method is sensitive to the extension length; therefore, by changing the size of the ellipse, the accuracy can be also changed. For extrapolation, we chose the square root function $\sqrt{a \xi^{2}+b \xi+c}$ which was used in [29], and from the extrapolated

Copyright (c) by SIAM. Unauthorized reproduction of this article is prohibited. 

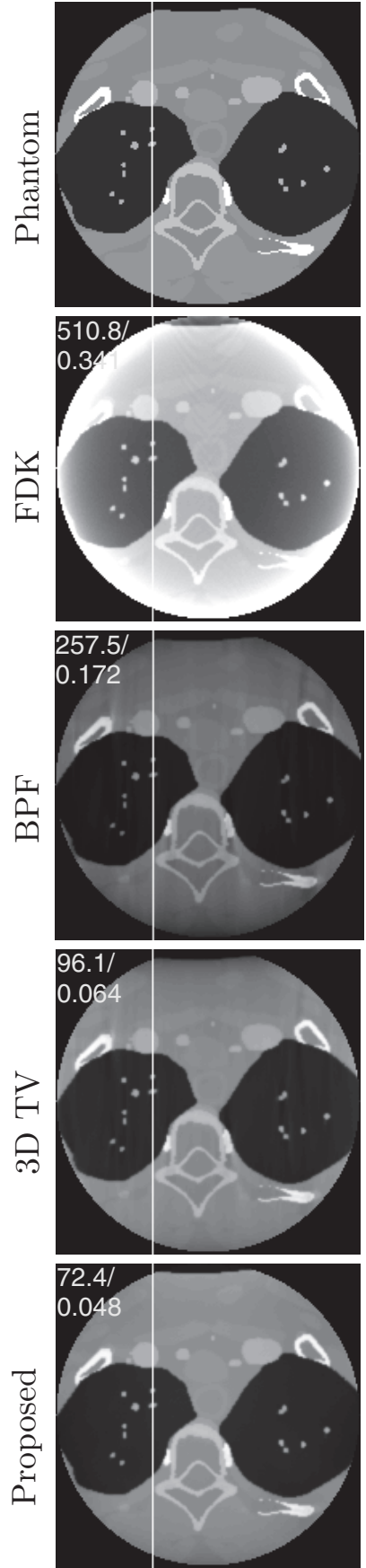

(a) 50
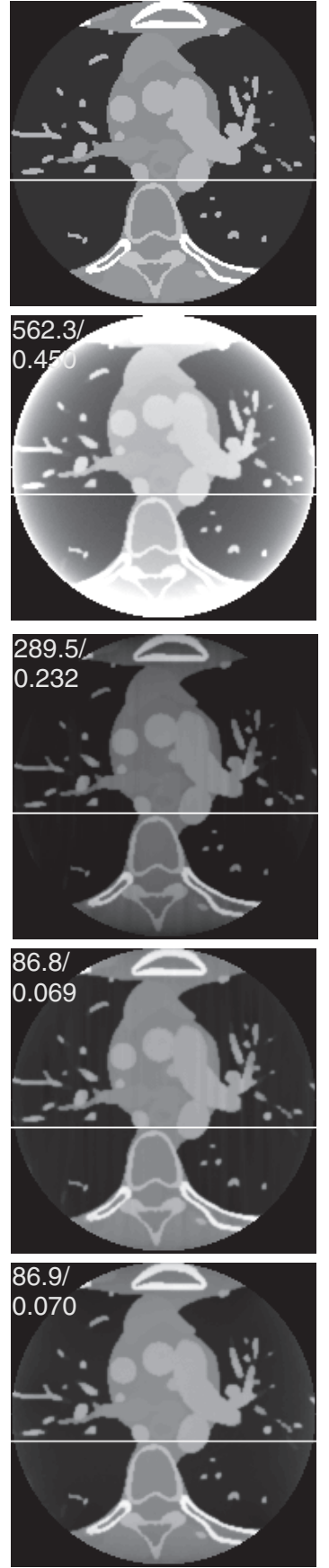

(b) 105
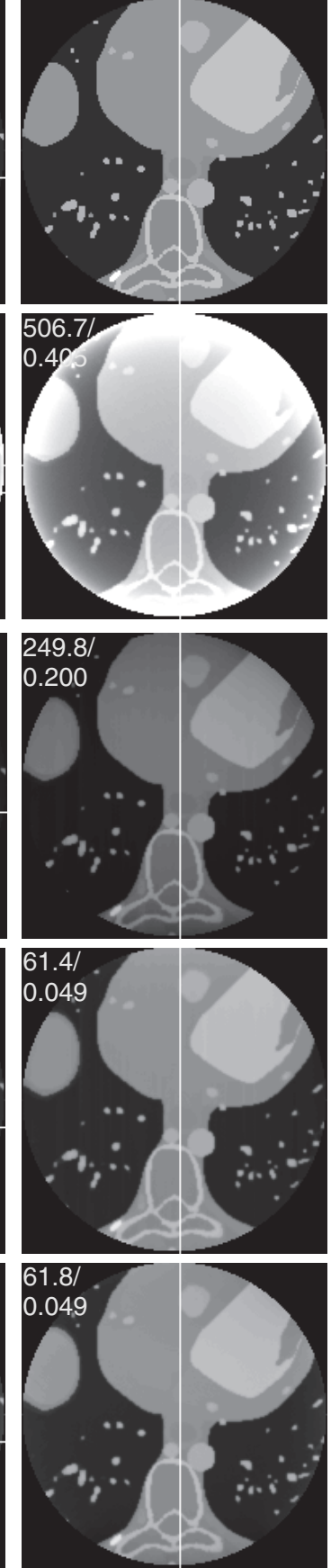

(c) 180

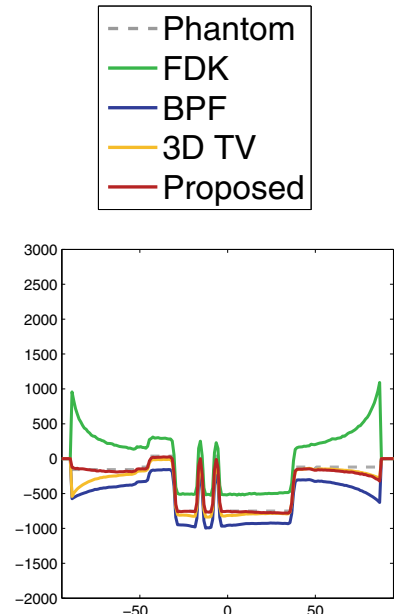

(a) 50

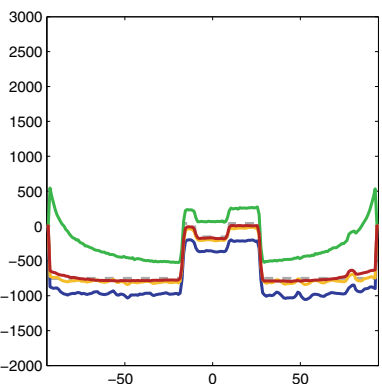

(b) 105

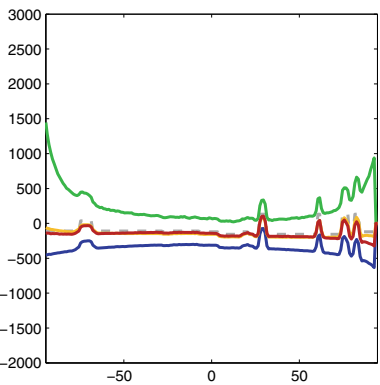

(c) 180

Figure 18. Reconstructed images by the truncated circular cone-beam simulation of the $3 D$ XCAT phantom. For the simulation, the $K$ of the $\mathrm{L}$ is 1 , and the iteration number of the POCS is 30. The $l$ and $u$ are 0 and 0.0309 , respectively, and the $\lambda$ is 0.02 , and $N$ is 20. The depth of the multiscale implementation is 2 . From the first to third columns, each column shows the slice image when the slice number is (a) 50, (b) 105, and (c) 180 when the midplane is the 160th slice, and each row is for the ideal phantom, FDK, BPF, 3D TV, and the proposed method. The last column shows the cut views indicated by the white line on the images. The number written at the corner of the reconstructed image shows the RMSE and NRMSE in the HU scale.

Copyright (C) by SIAM. Unauthorized reproduction of this article is prohibited. 

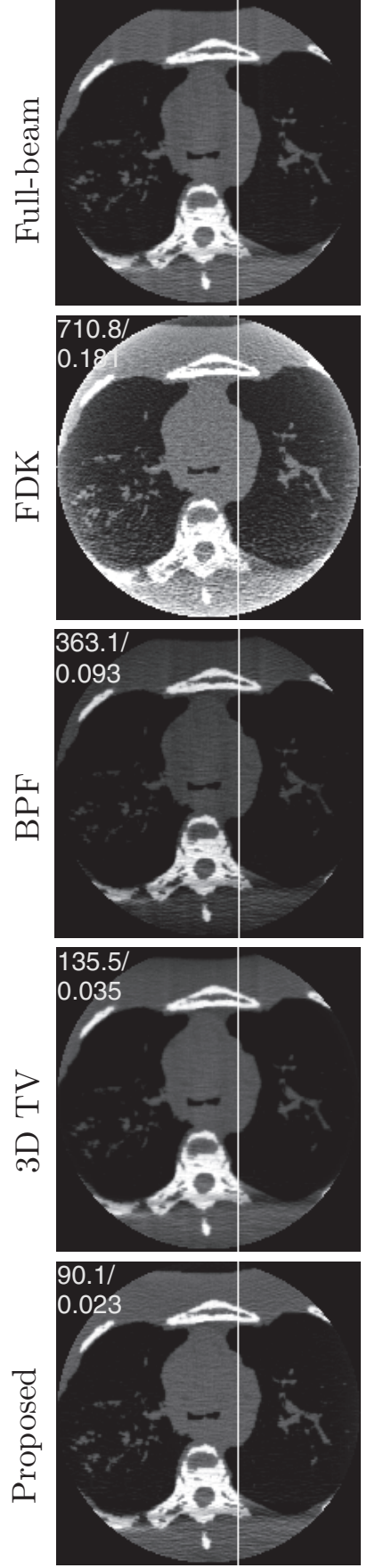

(a) Transverse
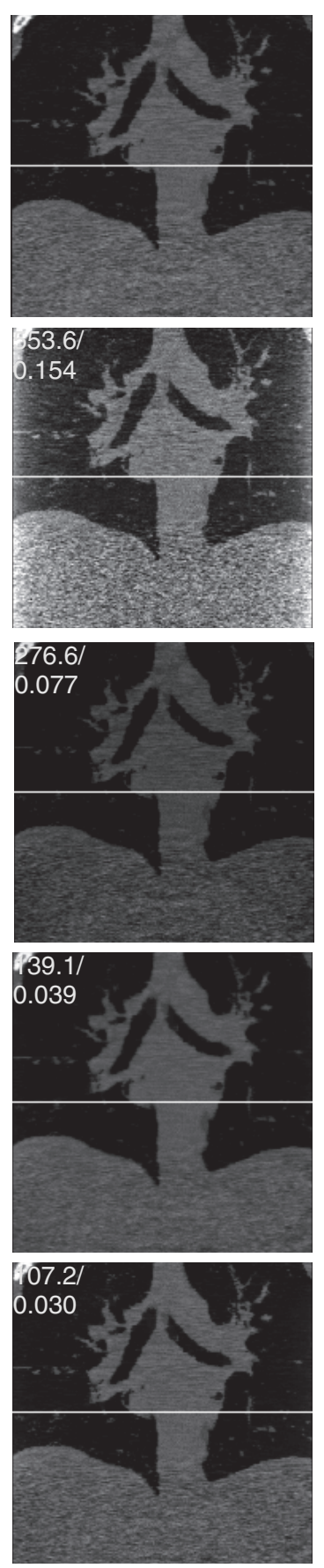

(b) Coronal
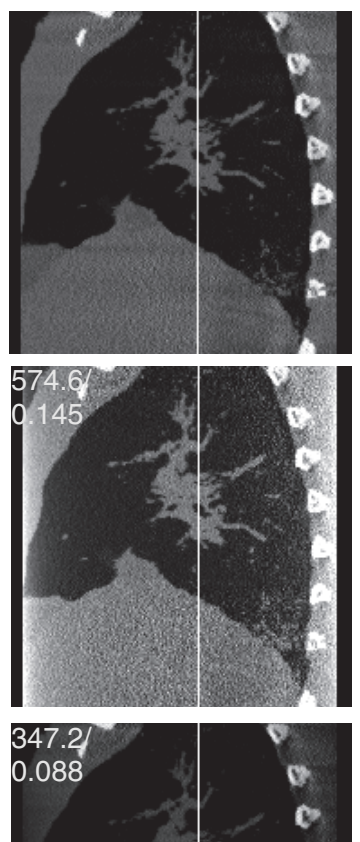

v.
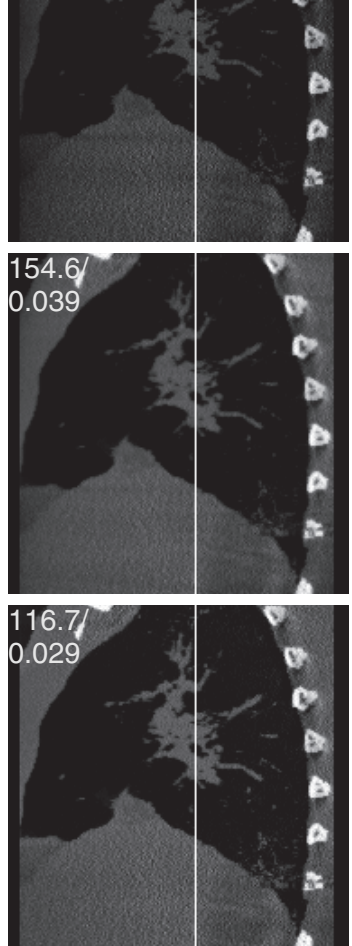

(c) Sagittal
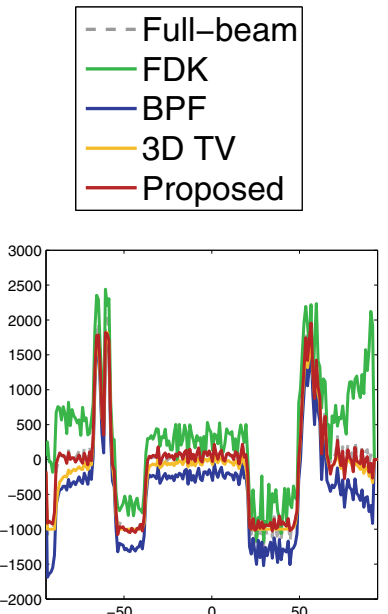

(a) Transverse

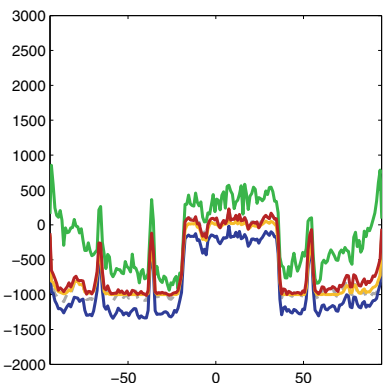

(b) Coronal

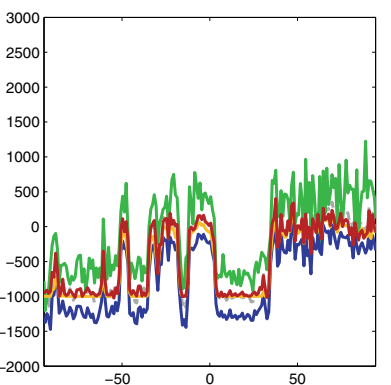

(c) Sagittal

Figure 19. Reconstructed images by the truncated circular cone-beam simulation of the $3 D$ inner organ phantom with noise. For the simulation, the $K$ of the $\mathrm{L}$ is 1 , and the iteration number of the POCS is 30 . The $l$ and $u$ are 0 and 0.0604, respectively, and the $\lambda$ is 0.01, and $N$ is 20. The depth of the multiscale implementation is 2. From the first to third columns, each column shows the same (a) $Z=100$, (b) $Y=260$, and (c) $X=220$ when the midplane is $Z=160$, and each row is for full-beam BPF, FDK, BPF, 3D TV, and the proposed method. The last column shows the cut views indicated by the white line on the images. The number written at the corner of the reconstructed image shows the RMSE and NRMSE in the HU scale. 
Table 4

Computational time (seconds) for circular cone-beam CT when $T R=65.8 \%$ and depth $=2$.

\begin{tabular}{lrr}
\hline & 3D TV & Proposed \\
\hline Vertical directional BPF & & 14.3 \\
Vertical directional multiscale reconstruction & & 33.4 \\
Horizonal directional BPF & & 13.4 \\
Horizontal directional multiscale reconstruction & & 33.4 \\
Spectral blending & & 2.1 \\
\hline Total (320 slices) & 1274.4 & 96.6 \\
Slices/sec & 0.251 & 3.313 \\
\hline
\end{tabular}

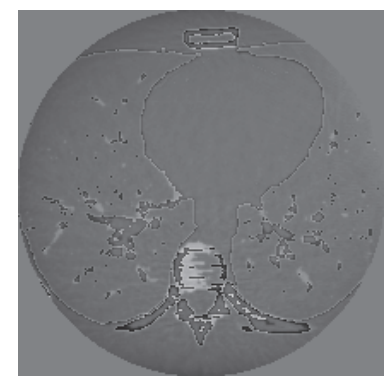

(a) Extrapolated FBP

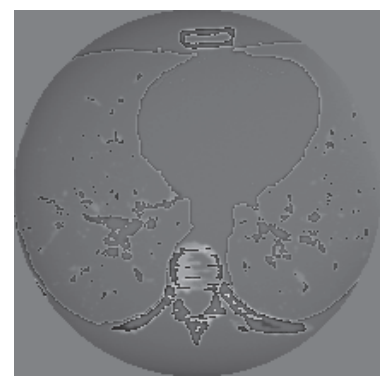

(b) Extrapolated BPF

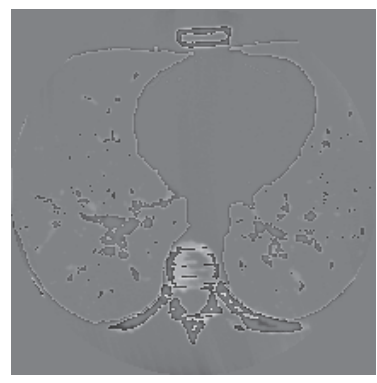

(c) Proposed

Figure 20. Error images from the extrapolated projection by FBP, BPF, and the proposed method when $L_{\text {extension }}=140$.

sinogram, the object was reconstructed by FBP and BPF. Figure 20 shows the error images of the 3D inner organ phantom by extrapolated FBP, extrapolated BPF, and the proposed method when $L_{\text {extension }}$ was $140 \mathrm{~mm}$, and all images are displayed with the same gray-scale map. Figures 20(a)-(c) have a similar high frequency error, but the low frequency error appears only in Figure 20(a)-(b). Figure 21 represents the NRMSE of the reconstructed result by extrapolated FBP, extrapolated $\mathrm{BPF}$, and the proposed method as the $L_{\text {extension }}$ increases. When the $L_{\text {extension }}$ was $60 \mathrm{~mm}$, the errors by all methods were similar, but as the $L_{\text {extension }}$ increased, the extrapolated methods had much bigger errors than that of the proposed method. As shown in Figures 20 and 21, our proposed method is more stable than the extrapolation methods.

5.2. Downsampling factor. Note that if the low frequency signal $f_{L}(x)$ is band-limited with a bandwidth less than $\omega_{0}$, then the corresponding DBP data $g_{L}=\mathcal{H} f_{L}$ should have the same bandwidth. However, for the truncated DBP signal, the converse is not generally true. More specifically, if we apply a low-pass filter to $w(x) g(x)$ to obtain $g_{L}(x)$ whose bandwidth is $\omega_{0}$, the recovered signal $f_{L}(x)$ from the truncated Hilbert transform under 1D TV penalty does not guarantee recovery of all the frequency content of $f(x)$ up to $\omega_{0}$. This is because our reconstruction method is nonlinear due to the generalized TV penalty to deal with data truncation. Consequently, the residual signal $g_{H}(x)=g(x)-\mathcal{H} f_{L}(x)$ should still have a lower frequency content below $\omega_{0}$. This indicates that the bandwidth $\omega_{0}$ should be determined conservatively compared to the linear recovery case. As a rule of thumb, we found

Copyright $\odot$ by SIAM. Unauthorized reproduction of this article is prohibited. 


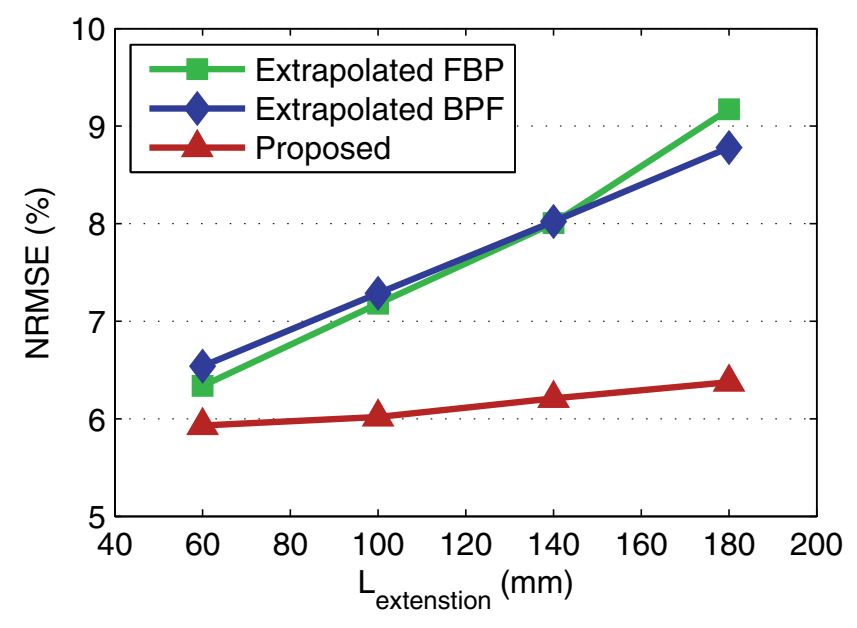

Figure 21. NRMSE value changes with respect to $L_{\text {extension }}$.

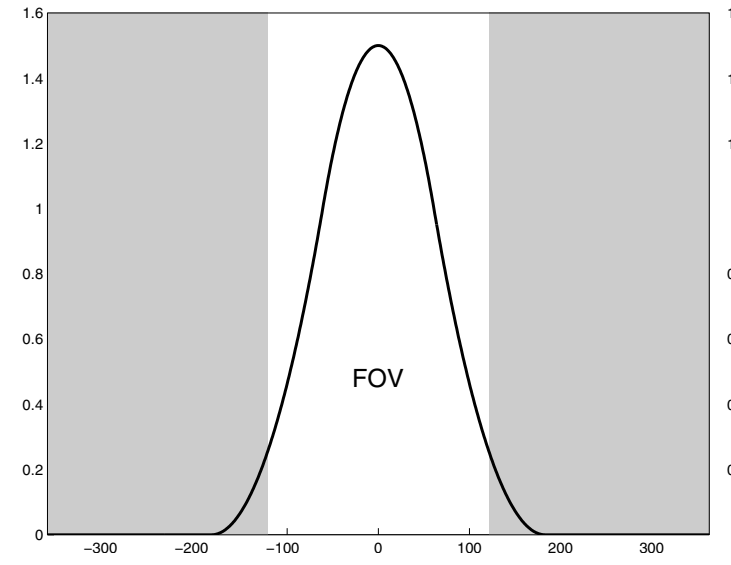

(a)

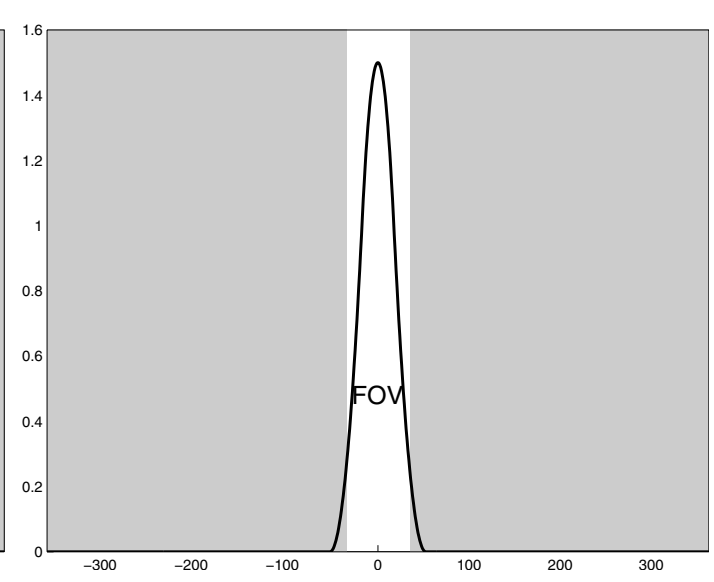

(b)

Figure 22. Window for interior tomography $w(x)$. (a) is for the large ROI case, and (b) is for the small ROI case.

that a bandwidth of $-65 \mathrm{~dB}$ is sufficient enough for our purpose of determining the cut-off frequency $\omega_{0}$.

Furthermore, to suppress the spectral overlap between the window $w(x)$ and $g_{H}(x)$, we can use a higher order window with a faster decay rate. Accordingly, we used a third order spline window, as shown in Figure 22. The advantage of using a third order spline rather than a first or second order spline is that the decay of its spectrum is faster.

Figures 23(a)-(b) show the reconstruction results with a large and small ROI, respectively; and Figures 23(c)-(d) are the spectrum of $w(x)$ and $g_{H}(x)$ for the large and small ROIs, respectively. Because the size of the window varies with the size of the ROI, the bandwidth of the window spectrum varies with the ROI, too. More specifically, a larger $w(x)$ has a smaller bandwidth. Accordingly, if we set the cut-off frequency $\omega_{0}$ to a bandwidth of $-65 \mathrm{~dB}$, the cut- 


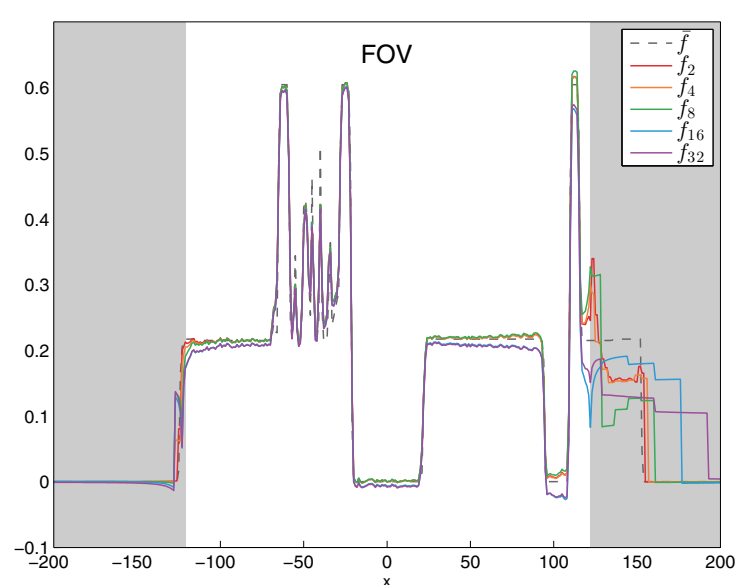

(a)

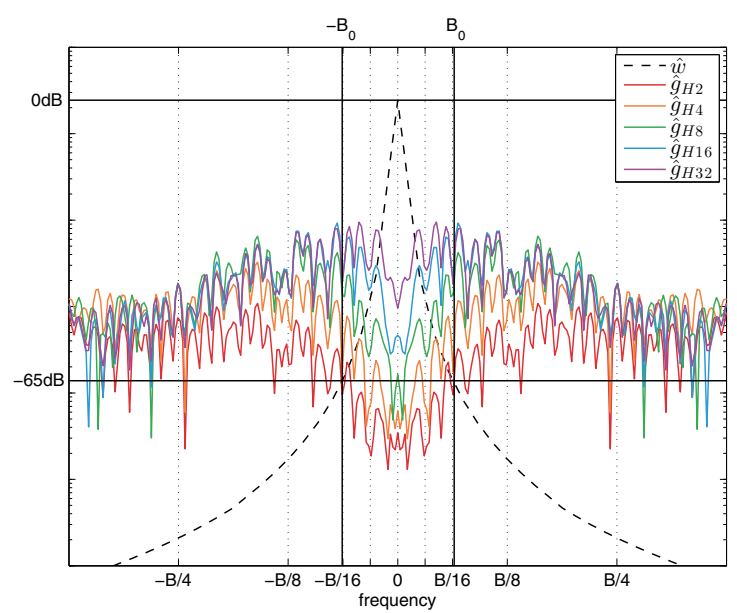

(c)

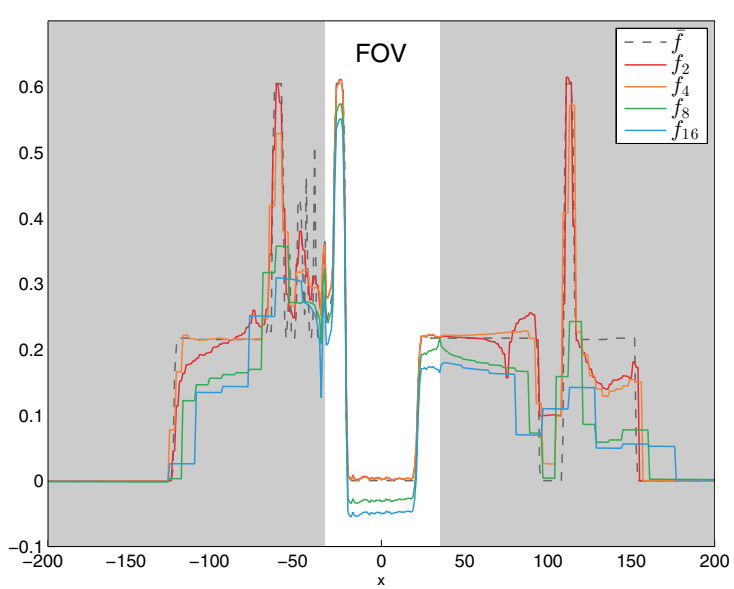

(b)

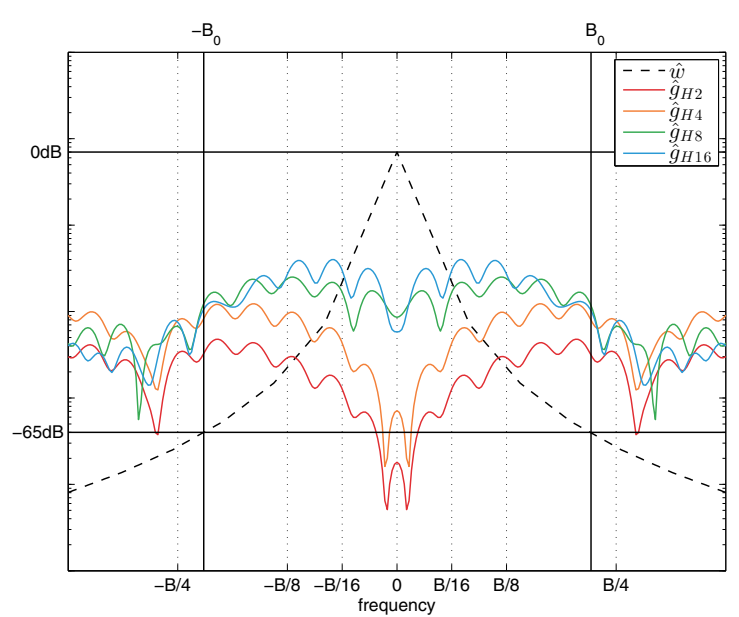

(d)

Figure 23. Interior tomography reconstruction results with various downsampling rates for two kinds of window sizes. Reconstructed $f(x)$ from (a) a large ROI and (b) a smaller ROI. The spectrum $\hat{w}(\omega)$ and $\hat{g}_{H}(\omega)$ for (c) a large ROI and (d) a smaller ROI. The subscript numbers after $f$ and $\hat{g}_{H}$ represent the downsampling rate. In (c)-(d), B refers to the Nyquist sampling rate of the original DBP data.

off frequency $\omega_{0}$ of a large ROI is smaller than that of the small ROI shown in Figure 23(c)-(d). In other words, in the case of a large ROI, we can perform more aggressive downsampling of the DBP signal for low frequency reconstruction. In Figure 23(c), the cut-off frequency is about $B / 16$, which means the maximum downsampling rate will be 16 , and in Figure 23(d), the cut-off frequency is larger than $B / 4$, which means the maximum downsampling rate will be 4. Accordingly, $f(x)$ can be reconstructed well with a downsampling rate of up to 16 in Figure 23(a), but in Figure 23(b), a downsampling rate of only up to 4 can produce the correct reconstruction. 
5.3. Role of spectral blending. The spatially invariant spectral blending scheme to deal with the missing frequency region can be equally applied for FBP or BPF types of algorithms. In fact, Pack et al. [25] applied a similar spectral blending idea for the FBP-type algorithm even though the detailed Fourier domain analysis was not fully developed in their original work.

The proposed interior tomography algorithm for 2D fan-beam CT or 3D helical cone-beam $\mathrm{CT}$ is performed on the actual chord (or PI) lines, so no missing frequency components for the chord lines exist, and the exact reconstruction is possible at the chord lines. However, in the reconstructions on the virtual chord lines from the circular cone-beam geometry, the spectral blending is required to minimize the missing frequency region. Figure 24 shows the results from the proposed method with and without spectral blending, respectively. Although the profiles for all results seem to be reconstructed well, the reconstruction using the multiscale interior tomography in either the horizontal or the vertical direction exhibits streaking artifacts along the filtering directions. However, the streaking artifacts were reduced by applying spectral blending, which confirms that spectral blending minimizes the missing frequency regions.

6. Conclusion. The existing iterative method of interior tomography based on $2 \mathrm{D}$ or $3 \mathrm{D}$ TV penalties is computationally too expensive to be used clinically because it requires multiple projections and backprojections. To overcome the disadvantage of the existing approaches, in our previous work, we proposed a $1 \mathrm{D}$ generalized TV seminorm penalty that is more relaxed but still sufficient to guarantee perfect recovery with significantly reduced computational complexity.

To further accelerate the algorithm, this paper proposed a multiscale interior tomography by exploiting the Bedrosian theorem. Thanks to the Bedrosian theorem, high frequency components of an object can be reconstructed analytically by the Hilbert transform; thus, only for the low frequency reconstruction, computationally expensive iterative reconstruction was performed at a very coarse resolution, which significantly reduced the computational complexity. Moreover, our TV modeling is only for the low frequency signal, which is more practical in capturing high frequency details that cannot be modeled by the generalized TV.

We further demonstrated that the DBP data from circular cone-beam data have a similar Hilbert transform relationship on virtual chord lines, but the content is different because of the missing frequency regions. To deal with additional cone-beam artifacts from incomplete acquisition geometry, we provided a novel analysis of the missing frequency region using Fourier domain analysis. Based on the analysis, a spectral blending scheme that weights the reconstruction from two orthogonal filtering directions was proposed. Accordingly, our algorithm for circular trajectory consisted of a two-step reconstruction procedure: first the multiscale interior tomography algorithm with 1D TV penalty on two orthogonal virtual chord lines and then the spectral blending of two reconstructions from the horizontal and vertical filtering directions.

The results of the numerical experiments showed that the proposed algorithm can produce a high quality reconstruction for both $2 \mathrm{D}$ fan-beam $\mathrm{CT}$, 3D helical cone-beam $\mathrm{CT}$, and $3 \mathrm{D}$ circular cone-beam $\mathrm{CT}$, and its computational time can be significantly reduced down to a practical level. Moreover, the spectral blending approach significantly reduced cone-beam and missing frequency artifacts with minimal increases in the computational complexity.

Copyright (c) by SIAM. Unauthorized reproduction of this article is prohibited. 

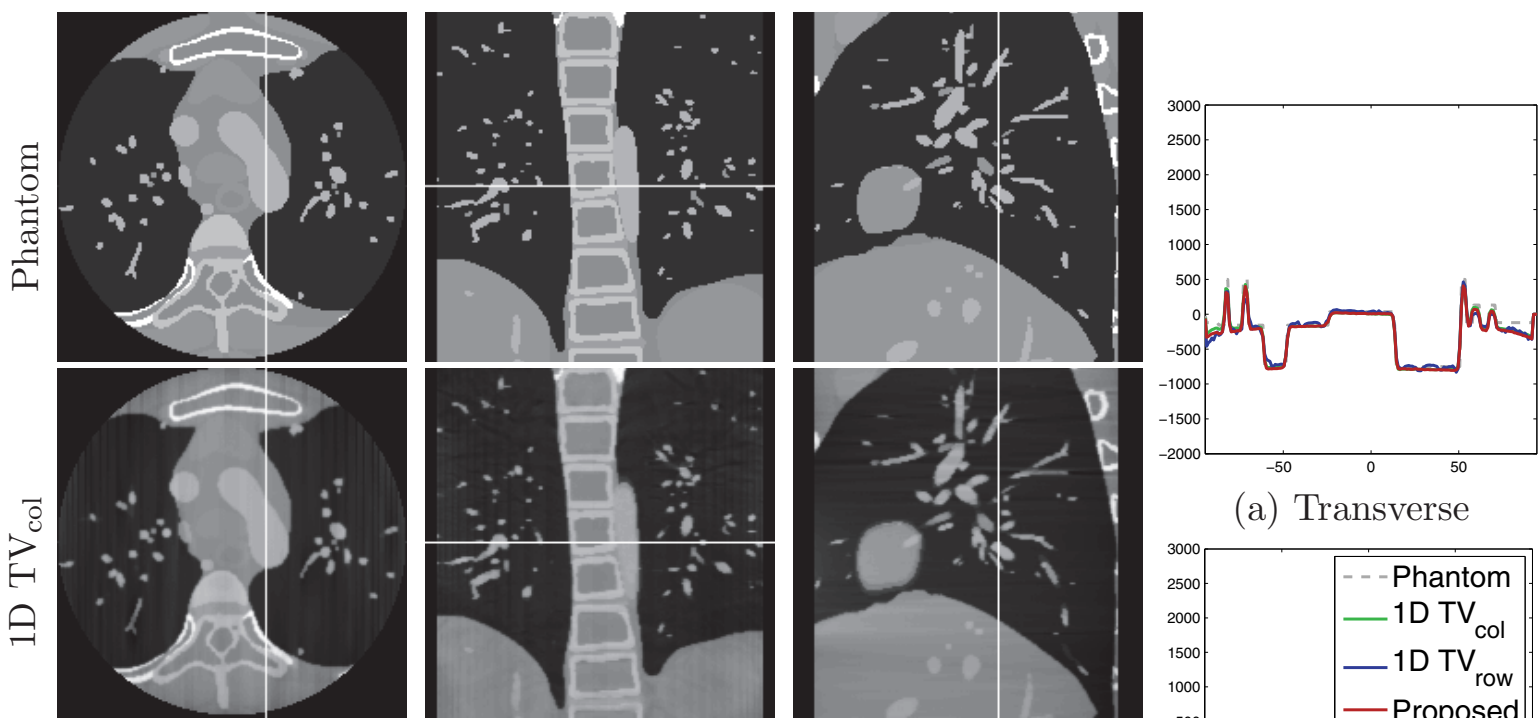

(a) Transverse
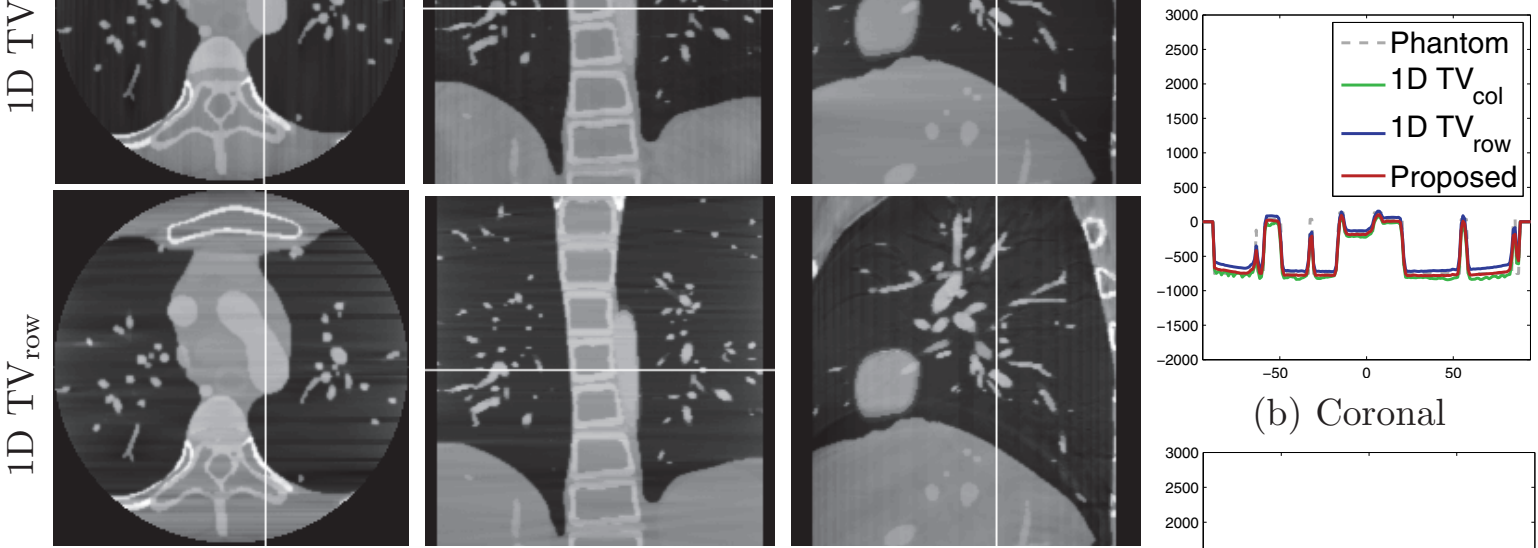

(b) Coronal

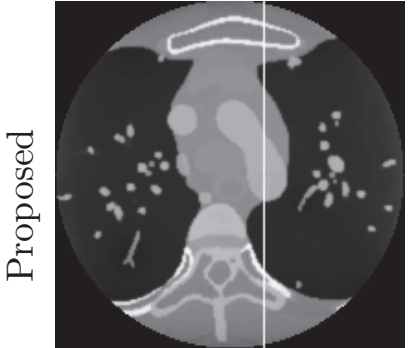

(a) Transverse

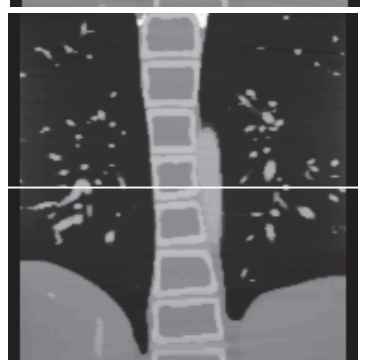

(b) Coronal

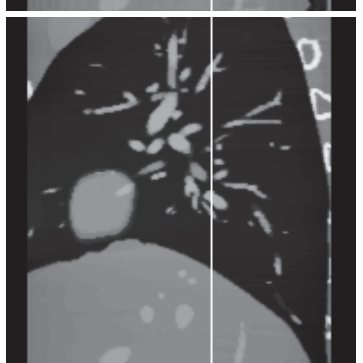

(c) Sagittal

Figure 24. 3D inner organ phantom reconstructed results from the proposed method with and without spectral blending. From the first to third columns, each column shows the slice image when the slice number is (a) $Z=90$, (b) $Y=290$, and (c) $X=210$ when the midplane is $Z=160$, and each row is for the ideal phantom, $1 D T V_{\mathrm{col}}, 1 D T V_{\mathrm{row}}$, and the proposed method. The last column shows the cut views indicated by the white line on the images.

\section{Appendix A. Proof of Theorem 2.2.}

Proof. Consider an axial view of the circular trajectory in Figure 6(a). Among the various choices of virtual chord lines, without loss of generality, we consider the horizontal direction from two virtual source locations $\mathbf{a}_{v}\left(\lambda_{1}^{-}\right)$and $\mathbf{a}_{v}\left(\lambda_{1}^{+}\right)$. In this case, we have

$$
\boldsymbol{\alpha}\left(\mathbf{x}, \lambda_{1}^{-}\right)=c^{-}\left[\begin{array}{lll}
x+\sqrt{R^{2}-y^{2}} & 0 & z
\end{array}\right]^{T}, \quad \boldsymbol{\alpha}\left(\mathbf{x}, \lambda_{1}^{+}\right)=c^{+}\left[\begin{array}{lll}
x-\sqrt{R^{2}-y^{2}} & 0 & z
\end{array}\right]^{T},
$$

Copyright (C) by SIAM. Unauthorized reproduction of this article is prohibited. 
where $c^{-}$and $c^{+}$denote normalization constants to make the corresponding vector unit norm. Accordingly, $\operatorname{sgn}(\boldsymbol{\alpha} \cdot \boldsymbol{\omega})$ can be expressed as

$$
\begin{aligned}
& \operatorname{sgn}\left(\boldsymbol{\alpha}\left(\mathbf{x}, \lambda_{1}^{-}\right) \cdot \boldsymbol{\omega}\right)=\operatorname{sgn}\left(\left(x+\sqrt{R^{2}-y^{2}}\right) \omega_{x}+z \omega_{z}\right), \\
& \operatorname{sgn}\left(\boldsymbol{\alpha}\left(\mathbf{x}, \lambda_{1}^{+}\right) \cdot \boldsymbol{\omega}\right)=\operatorname{sgn}\left(\left(x-\sqrt{R^{2}-y^{2}}\right) \omega_{x}+z \omega_{z}\right) .
\end{aligned}
$$

Then, the corresponding DBP data can be represented as

$$
g(\mathbf{x})=\frac{1}{(2 \pi)^{3}} \int d \boldsymbol{\omega} e^{j \mathbf{x} \cdot \boldsymbol{\omega}} \hat{f}(\boldsymbol{\omega}) j \sigma\left(\mathbf{x}, \boldsymbol{\omega}, \lambda_{1}^{-}, \lambda_{1}^{+}\right),
$$

where $\sigma\left(\mathbf{x}, \boldsymbol{\omega}, \lambda_{1}^{-}, \lambda_{1}^{+}\right)$is defined by

$$
\begin{aligned}
\sigma\left(\mathbf{x}, \boldsymbol{\omega}, \lambda_{1}^{-}, \lambda_{1}^{+}\right) & =\frac{1}{2}\left[\operatorname{sgn}\left(\boldsymbol{\alpha}\left(\mathbf{x}, \lambda_{1}^{-}\right) \cdot \boldsymbol{\omega}\right)-\operatorname{sgn}\left(\boldsymbol{\alpha}\left(\mathbf{x}, \lambda_{1}^{+}\right) \cdot \boldsymbol{\omega}\right)\right] \\
& =\frac{1}{2}\left[\operatorname{sgn}\left(\omega_{x}-A\right)+\operatorname{sgn}\left(\omega_{x}-B\right)\right],
\end{aligned}
$$

where

$$
A=-\frac{z \omega_{z}}{x+\sqrt{R^{2}-y^{2}}}, \quad B=\frac{z \omega_{z}}{\sqrt{R^{2}-y^{2}}-x} .
$$

Accordingly, for $z \omega_{z} \geq 0, \sigma\left(\mathbf{x}, \omega, \lambda_{1}^{-}, \lambda_{1}^{+}\right)=0$ when $A<\omega_{x}<B$. Similarly, for $z \omega_{z}<0$, $\sigma\left(\mathbf{x}, \boldsymbol{\omega}, \lambda_{1}^{-}, \lambda_{1}^{+}\right)=0$ when $B<\omega_{x}<A$. Otherwise, $\sigma\left(\mathbf{x}, \boldsymbol{\omega}, \lambda_{1}^{+}, \lambda_{1}^{-}\right)=\operatorname{sgn}\left(\omega_{x}\right)$. This leads to the following:

$$
\sigma\left(\mathbf{x}, \boldsymbol{\omega}, \lambda_{1}^{+}, \lambda_{1}^{-}\right)= \begin{cases}0 & \text { if }\left(\omega_{x}, \omega_{y}, \omega_{z}\right) \in \mathcal{N}(z), \\ \operatorname{sgn}\left(\omega_{x}\right) & \text { otherwise }\end{cases}
$$

where the missing frequency set $\mathcal{N}(z)$ on a slice at $z$ is given by

$$
\begin{aligned}
\mathcal{N}(z) & =\left\{\min \{A, B\} \leq \omega_{x} \leq \max \{A, B\}\right\} \\
& =\left\{\left(\omega_{x}, \omega_{y}, \omega_{z}\right) \mid-\frac{1}{x+\sqrt{R^{2}-y^{2}}} \leq \frac{\omega_{x}}{z \omega_{z}} \leq \frac{1}{\sqrt{R^{2}-y^{2}}-x}\right\},
\end{aligned}
$$

where the last equality comes from considering both $z \omega_{z}>0$ and $z \omega_{z}<0$. For $z=0$, the missing frequency region is reduced to a null set. Accordingly, (A.2) can be simplified as

$$
g(\mathbf{x})=\frac{1}{2 \pi} \int_{-\infty}^{\infty} d \omega_{x} \hat{\phi}\left(\omega_{x}, y, z\right) j \operatorname{sgn}\left(\omega_{x}\right) e^{j x \omega_{x}},
$$

where

$$
\hat{\phi}\left(\omega_{x}, y, z\right):=\frac{1}{(2 \pi)^{2}} \int d \omega_{y} \int_{\omega_{z} \in\left\{\left(\omega_{x}, \omega_{y}, \omega_{z}\right) \notin \mathcal{N}(z)\right\}} d \omega_{z} \hat{f}\left(\omega_{x}, \omega_{y}, \omega_{z}\right) e^{j\left(y \omega_{y}+z \omega_{z}\right)} .
$$

Copyright $\odot$ by SIAM. Unauthorized reproduction of this article is prohibited. 
Now, the remaining part of the proof is to show that this holds for the e of the arbitrary chord line. This can be done simply by changing the expression in (A.4) as well as the integral representation using the virtual coordinate values $\left(x^{\prime}, y^{\prime}, z\right)$ defined in $(2.10)$ :

$$
\mathcal{N}(z)=\left\{\left(\omega_{x^{\prime}}, \omega_{y^{\prime}}, \omega_{z}\right) \in \mathbb{R} \mid-\frac{1}{x^{\prime}+\sqrt{R^{2}-\left(y^{\prime}\right)^{2}}} \leq \frac{\omega_{x^{\prime}}}{z \omega_{z}} \leq \frac{1}{\sqrt{R^{2}-\left(y^{\prime}\right)^{2}}-x^{\prime}}\right\}
$$

and

$$
g(\mathbf{x})=\frac{1}{2 \pi} \int_{-\infty}^{\infty} d \omega_{x^{\prime}} \hat{\phi}\left(\omega_{x^{\prime}}, y^{\prime}, z\right) j \operatorname{sgn}\left(\omega_{x^{\prime}}\right) e^{j \omega_{x^{\prime}} x^{\prime}}
$$

where

$$
\hat{\phi}\left(\omega_{x^{\prime}}, y^{\prime}, z\right):=\frac{1}{(2 \pi)^{2}} \int d \omega_{y^{\prime}} \int_{\omega_{z} \in\left\{\left(\omega_{x^{\prime}}, \omega_{y^{\prime}}, \omega_{z}\right) \notin \mathcal{N}(z)\right\}} d \omega_{z} \hat{f}\left(\omega_{x^{\prime}}, \omega_{y^{\prime}}, \omega_{z}\right) e^{j\left(y^{\prime} \omega_{y^{\prime}}+z \omega_{z}\right)}
$$

This concludes the proof.

\section{REFERENCES}

[1] S. Bartolac, R. Clackdoyle, F. Noo, J. Siewerdenen, D. Moseley, and D. Jaffray, A local shift-variant Fourier model and experimental validation of circular cone-beam computed tomography artifacts, Med. Phys., 36 (2009), pp. 500-512.

[2] A. Beck And M. Teboulle, Fast gradient-based algorithms for constrained total variation image denoising and deblurring problems, IEEE Trans. Image Process., 18 (2009), pp. 2419-2434.

[3] J. Brokish And Y. BREsler, Sampling requirements for circular cone beam tomography, in IEEE Nuclear Science Symposium Conference Record, 2006, Vol. 5, IEEE, Piscataway, NJ, 2006, pp. 2882-2884.

[4] A. V. Bronnikov, Cone-beam reconstruction by backprojection and filtering, J. Opt. Soc. Am. A, 17 (2000), pp. 1993-2000.

[5] R. Clackdoyle, F. Noo, J. Guo, and J. A. Roberts, Quantitative reconstruction from truncated projections in classical tomography, IEEE Trans. Nucl. Sci., 51 (2004), pp. 2570-2578.

[6] M. Courdurier, F. Noo, M. Defrise, And H. Kudo, Solving the interior problem of computed tomography using a priori knowledge, Inverse Problems, 24 (2008), 065001.

[7] S. Dale And P. Edholm, Inherent limitations in ectomography, IEEE Trans. Med. Imag., 7 (1988), pp. $165-172$.

[8] M. Defrise, F. Noo, R. Clackdoyle, and H. Kudo, Truncated Hilbert transform and image reconstruction from limited tomographic data, Inverse Problems, 22 (2006), pp. 1037-1053.

[9] F. Dennerlein, F. Noo, H. Schondube, G. Lauritsch, And J. Hornegger, A factorization approach for cone-beam reconstruction on a circular short-scan, IEEE Trans. Med. Imag., 27 (2008), pp. 887896.

[10] J. T. Dobbins III And D. J. Godfrey, Digital x-ray tomosynthesis: Current state of the art and clinical potential, Phys. Med. Biol., 48 (2003), pp. R65-R106.

[11] J. M. FAdili AND G. PEYRÉ, Total variation projection with first order schemes, IEEE Trans. Image Process., 20 (2011), pp. 657-669.

[12] P. Grangeat, Analyse d'un système d'imagerie $3 D$ par reconstruction à partir de radiographies $X$ en géométrie conique, Ph.D. thesis, ENST, Paris, 1987.

[13] W. HAN, H. Yu, AND G. WANG, A general total variation minimization theorem for compressed sensing based interior tomography, Int. J. Biomed. Imaging, 2009 (2009), 125871.

[14] J. Hsieh, E. Chao, J. Thibault, B. Grekowicz, A. Horst, S. McOlash, and T. J. Myers, Algorithm to extend reconstruction field-of-view, in IEEE International Symposium on Biomedical Imaging: Nano to Macro, 2004, Vol. 2, IEEE, Los Alamitos, CA, 2004, pp. 1404-1407.

Copyright $\odot$ by SIAM. Unauthorized reproduction of this article is prohibited. 
[15] X. Jin, A. Katsevich, H. Yu, G. Wang, L. Li, and Z. Chen, Interior tomography with continuous singular value decomposition, IEEE Trans. Med. Imag., 31 (2012), pp. 2108-2119.

[16] A. Katsevich, Theoretically exact filtered backprojection-type inversion algorithm for spiral CT, SIAM J. Appl. Math., 62 (2002), pp. 2012-2026.

[17] A. Katsevich AND A. TovBis, Finite Hilbert transform with incomplete data: Null-space and singular values, Inverse Problems, 28 (2012), 105006.

[18] E. Katsevich, A. Katsevich, And G. Wang, Stability of the interior problem with polynomial attenuation in the region of interest, Inverse Problems, 28 (2012), 065022.

[19] F. W. King, Hilbert Transforms, Vol. 2, Cambridge University Press, Cambridge, UK, 2009.

[20] E. Meyer, R. Raupach, M. Lell, B. Schmidt, and M. Kachelriess, Frequency split metal artifact reduction (FSMAR) in computed tomography, Med. Phys., 39 (2012), pp. 1904-1916.

[21] Y. Nesterov, A method of solving a convex programming problem with convergence rate $O\left(1 / k^{2}\right)$, Soviet Math. Dokl., 27 (1983), pp. 372-376.

[22] F. Noo, R. Clackdoyle, and J. D. Pack, A two-step Hilbert transform method for $2 D$ image reconstruction, Phys. Med. Biol., 49 (2004), pp. 3903-3923.

[23] J. D. PACK AND F. NoO, Cone-beam reconstruction using $1 D$ filtering along the projection of M-lines, Inverse Problems, 21 (2005), pp. 1105-1120.

[24] J. D. PACK, F. Noo, AND R. ClaCKdoyle, Cone-beam reconstruction using the backprojection of locally filtered projections, IEEE Trans. Med. Imag., 24 (2005), pp. 70-85.

[25] J. D. PACK, Z. Yin, K. ZENG, AND B. E. NetT, Mitigating cone-beam artifacts in short-scan CT imaging for large cone-angle scans, in Proceedings of the International Meeting on Fully 3D Image Reconstruction in Radiology and Nuclear Medicine, 2013, pp. 307-310.

[26] F. Peyrin, M. Amiel, And R. Goutte, Analysis of a cone beam x-ray tomographic system for different scanning modes, J. Opt. Soc. Am. A, 9 (1992), pp. 1554-1563.

[27] L. Ritschl, F. Bergner, C. Fleischmann, and M. Kachelriess, Improved total variation-based CT image reconstruction applied to clinical data, Phys. Med. Biol., 56 (2011), pp. 1545-1561.

[28] G. Shechter, Th. Köhler, A. Altman, and R. Proksa, The frequency split method for helical conebeam reconstruction, Med. Phys., 31 (2004), pp. 2230-2236.

[29] K. Sourbelle, M. Kachelriess, and W. A. Kalender, Reconstruction from truncated projections in CT using adaptive detruncation, Eur. Radiol., 15 (2005), pp. 1008-1014.

[30] K. C. Tam, G. Lauritsch, and K. Sourbelle, Filtering point spread function in backprojection conebeam CT and its applications in long object imaging, Phys. Med. Biol., 47 (2002), pp. 2685-2703.

[31] H. K. TuY, An inversion formula for cone-beam reconstruction, SIAM J. Appl. Math., 43 (1983), pp. 546552.

[32] J. P. WARD, M. LeE, J. C. Ye, AND M. Unser, Interior tomography using $1 D$ generalized total variation. Part I: Mathematical foundation, SIAM J. Imaging Sci., 8 (2015), pp. 226-247.

[33] X. H. YAN AND R. M. LEAHY, Derivation and analysis of a filtered backprojection algorithm for cone beam projection data, IEEE Trans. Med. Imag., 10 (1991), pp. 462-472.

[34] J. YANG, H. YU, M. JIANG, AND G. WANG, High-order total variation minimization for interior tomography, Inverse Problems, 26 (2010), 035013.

[35] H. Yu AND G. WAng, Compressed sensing based interior tomography, Phys. Med. Biol., 54 (2009), pp. 2791-2805.

[36] L. Yu, Y. Zou, E. Y. Sidky, C. A. Pelizzari, P. Munro, and X. Pan, Region of interest reconstruction from truncated data in circular cone-beam CT, IEEE Trans. Med. Imag., 25 (2006), pp. 869-881.

[37] B. Zhang AND G. L. ZENG, Two-dimensional iterative region-of-interest (ROI) reconstruction from truncated projection data, Med. Phys., 34 (2007), pp. 935-944.

[38] Y. Zou AND X. PAN, Exact image reconstruction on PI-lines from minimum data in helical cone-beam CT, Phys. Med. Biol., 49 (2004), pp. 941-959.

[39] Y. Zou AND X. PAN, Image reconstruction on PI-lines by use of filtered backprojection in helical cone-beam CT, Phys. Med. Biol., 49 (2004), pp. 2717-2731.

[40] Y. ZOU, X. PAN, AND E. Y. SIDKY, Image reconstruction in regions-of-interest from truncated projections in a reduced fan-beam scan, Phys. Med. Biol., 50 (2005), pp. 13-27.

Copyright ( $\odot$ by SIAM. Unauthorized reproduction of this article is prohibited. 